\title{
Differential diffusion effects, distributed burning, and local extinctions in high Karlovitz premixed flames
}

\author{
Simon Lapointe ${ }^{a, 1}$, Bruno Savard ${ }^{a}$, and Guillaume Blanquart ${ }^{b}$ \\ ${ }^{a}$ Graduate Aerospace Laboratories, California Institute of Technology, Pasadena, CA \\ 91125, USA \\ ${ }^{b}$ Mechanical Engineering Department, California Institute of Technology, Pasadena, CA \\ 91125, USA
}

\begin{abstract}
Direct numerical simulations of premixed $n$-heptane/air flames at different Karlovitz numbers are performed using detailed chemistry. Differential diffusion effects are systematically isolated by performing simulations with both non-unity and unity Lewis numbers. Different unburnt temperatures and turbulence intensities are used and their effects on the flame structure and chemical source terms are investigated. As the unburnt gases are preheated, the viscosity ratio across the flame is reduced and the Karlovitz number at the reaction zone is increased. The increase in turbulence intensity suppresses differential diffusion effects on the flame structure (i.e. species dependence on temperature). However, differential diffusion effects on the chemical source terms are still noticeable even at the highest Karlovitz number simulated. Simulations with differential diffusion effects exhibit lower mean fuel consumption and heat release rates than their unity Lewis number counterparts. However, the difference is reduced as the reaction zone Karlovitz number is increased. Transition to distributed burning is characterized by a broadening of the reaction zone resulting from enhanced turbulent mixing. Local extinctions in the burning rate are observed only in non-unity Lewis number simulations and their probability decreases at


high Karlovitz numbers. These results highlight the importance of using the reaction zone Karlovitz number to investigate the effect of turbulence on the chemical source terms and to compare flames at different unburnt temperatures.

Key words: turbulent premixed flames, differential diffusion effects, distributed burning, broken reaction zone, high Karlovitz

\section{Introduction}

In the context of Peters' regime diagram [1,2], the interaction between a premixed flame and turbulence is characterized by a velocity ratio $u^{\prime} / S_{L}$ and a length ratio $l / l_{F}$, where $u^{\prime}$ is the turbulence fluctuations, $l$ is the integral length scale, $S_{L}$ is the unstretched laminar flame speed, and $l_{F}$ is the laminar flame thickness $l_{F}=\left(T_{b}-\right.$ $\left.T_{u}\right) / \max (|\nabla T|)$. The relevant dimensionless groups are the Reynolds, Karlovitz, and Damkohler numbers, defined in the present study as

$$
\operatorname{Re}_{l}=\frac{u^{\prime} l}{\nu}, \mathrm{Ka}=\frac{l_{F}}{S_{L}} \sqrt{\frac{u^{\prime 3}}{\nu l}}, \mathrm{Da}=\frac{l / u^{\prime}}{l_{F} / S_{L}}
$$

where $\nu$ is the kinematic viscosity. Practical premixed combustion devices operate at elevated unburnt temperatures and pressures, under high turbulence intensities, and use heavy hydrocarbon-based fuels. These applications fall in the thin or broken/distributed reaction zones regimes, which are characterized by a large Karlovitz number [3].

Experiments are difficult to conduct at high Karlovitz numbers and a limited number of them are available in the literature [4-8]. Similarly, few direct numerical

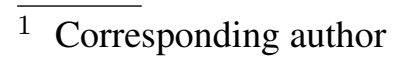

Email address: slapoint@caltech.edu (S. Lapointe) 
simulations (DNS) of turbulent premixed flames with detailed chemistry have been performed in the thin and broken/distributed reaction zones regimes [9-15]. In an effort to reduce computational costs, studies have been performed with simplified chemistry, such as one-step chemical models $[16,17]$. However, detailed kinetic modeling remains necessary to study the impact of high-speed turbulence on the chemistry especially in the thin and broken/distributed reaction zones regimes.

High Karlovitz number flames are fundamentally different from low Karlovitz number flames because the turbulence penetrates inside and disturbs the reaction zone. From a theoretical and modeling point of view, three aspects need to be investigated: namely the impact of differential diffusion, the distributed nature of the reaction zone, and possible local extinction events. The present numerical work focuses on these three critical aspects by considering a heavy hydrocarbon fuel, $n$-heptane. In contrast, previous numerical simulations at high Karlovitz numbers using detailed chemistry considered mostly simple fuels such as hydrogen [11$13,18]$, methane $[14,15,18]$, and propane [14]. Recent experimental work $[8,19]$ studied $n$-heptane/air combustion (and other heavy hydrocarbon fuels) in turbulent premixed flames. Since most transportation fuels are composed of large linear alkanes, cyclo-alkanes, iso-alkanes, and aromatic species, the study of all of these types of hydrocarbons is of interest. The present work focuses on a linear alkane, but the study of cyclo-alkanes, iso-alkanes, and aromatics should be the subject of future work as their chemistry is known to differ significantly from that of simpler fuels such as methane and propane.

Aspden et al. [11] studied the interaction of lean premixed hydrogen flames with turbulence over a wide range of Karlovitz numbers. Distributed burning was observed at high Karlovitz numbers $(\mathrm{Ka} \approx 4200)$ and global extinction did not occur. The distributed flame was characterized by a broad mixing region, similar to tur- 
bulent mixing of a passive scalar. Turbulent mixing was dominant over molecular mixing and differential diffusion effects were again significantly reduced. Aspden et al. [14] investigated Lewis number effects in distributed hydrogen $\left(\operatorname{Le}_{\text {fuel }}<1\right)$, methane $\left(\operatorname{Le}_{\text {fuel }} \sim 1\right)$, and propane flames $\left(\operatorname{Le}_{\text {fuel }}>1\right)$. It was shown that distributed burning led to an enhanced burning rate in low Lewis number flames while the burning rates of methane and propane flames remained closer to their laminar values.

DNS of premixed $n$-heptane-air turbulent flames close to the transition between the thin/broken reaction zones were recently presented in Savard et al. [20,21]. To better analyze differential diffusion effects, two simulations were performed, one with unity Lewis numbers and one with non-unity Lewis numbers. The flame structure (defined as the dependence of species mass fractions on temperature) of the unity Lewis number flame was shown to be very similar to that of a one-dimensional, flat flame, suggesting that turbulence has a very limited impact on the flame in temperature space, in the absence of differential diffusion. On the other hand, the structure of the non-unity Lewis number flame was affected more substantially by turbulence. It was argued that turbulence affects the flame structure through an effective Lewis number [2,22]. At high turbulence levels (i.e. high Karlovitz number) turbulence reduces differential diffusion effects consistently with the results of Aspden et al. $[11,14]$. These effects were almost suppressed in the preheat zone, but were still present at the reaction zone while the reaction zone remained thin. The fuel consumption rate exhibited significant fluctuations (both for the unity and non-unity Lewis number simulations) and local extinctions were observed in the non-unity Lewis number case.

These studies provided valuable, yet still qualitative insight in the behavior of flames in the distributed burning regime. There is still a need for quantifying the 
impact of differential diffusion effects and distributed burning on chemical source terms fluctuations and local extinctions.

In addition, it is important to underline that most of these studies have been performed at ambient temperatures while most combustion devices operate at higher unburnt temperatures. An increase in the unburnt temperature will lead to a reduction in the viscosity ratio across the flame. As the Kolmogorov length scale depends on the viscosity, small-scale turbulent structures are then expected to penetrate further in the reaction zone. Considering a fixed velocity ratio $u^{\prime} / S_{L}$ and length ratio $l / l_{F}$, the change in viscosity ratio will lead to an increased Karlovitz number at the reaction zone while the Karlovitz number in the unburnt gases would not change. To take these effects into account, it is more adequate to use the reaction zone Karlovitz number [1]:

$$
\mathrm{Ka}_{\delta}=\frac{\delta_{F, \mathrm{lam}}^{2}}{\eta_{\delta}^{2}}
$$

where $\delta_{F, \text { lam }}$ is the laminar reaction zone thickness and $\eta_{\delta}$ denotes the value of the Kolmogorov length scale at the reaction zone (taken at $T_{\text {peak }}$, the temperature corresponding to the peak value of the fuel consumption rate in the flame). Equation (2) is preferred in the present work to distinguish between the different unburnt temperatures.

The present study builds upon previous studies by Savard et al. [20,21] and aims at investigating the transition from the thin to broken/distributed reaction zone regimes in premixed flames with an engine-relevant, heavy hydrocarbon fuel, namely $n$-heptane. In contrast to [20,21], turbulent flames are studied over a range of Karlovitz numbers, covering the thin reaction zone and the distributed burning regimes. More precisely, the Karlovitz number at the reaction zone is increased incrementally using different turbulence intensities and unburnt temperatures. The 
focus is placed on three main objectives.

- Understand and quantify differential diffusion effects on burning rate and heat release.

- Identify the transition to distributed burning and characterize the resulting reaction zone.

- Quantify and explain the occurence of local extinctions at increasing turbulence intensities.

In Section 2, the computational methodology is reviewed. An overview of the results is presented in Section 3. The three objectives are addressed in sections 4, 5, and 6. Section 4 focuses on the role of differential diffusion on the fuel consumption and heat release rates; the transition to distributed burning is quantified in Section 5; and Section 6 describes local extinctions and their connection with differential diffusion effects. Section 7 highlights the importance of the Karlovitz number definition. Finally, the results are summarized and their implications for combustion modeling are discussed in Section 8.

\section{Computational methodology}

The direct numerical simulations performed in this study follow the methodology presented in Savard et al. [20] and Savard and Blanquart [21]. For completeness, the numerical framework is briefly reviewed here. A total of nine simulations have been performed. The parameters for each simulation are provided in Table 1. 


\subsection{Flow configuration}

The same flow configuration is used for all the simulations and is illustrated in Fig. 1. It was chosen to achieve statistically-planar, three-dimensional, freely propagating flames without mean shear and mean curvature effects. Inflow/outflow boundary conditions are used in the $x$ direction and periodic boundary conditions are used in the $y$ and $z$ directions. The domain size is $11 L \times L \times L$ in $x, y$, and $z$ respectively. The relation of the domain width, $L$, to the integral length scale is discussed in Section 2.4. The simulations can be run for an unbounded time, allowing to reach a statistically-stationary state. To avoid negative inlet velocities, the unburnt gases are introduced with a low turbulent kinetic energy (TKE). Since the flow configuration lacks generation of turbulence due to large scale flow straining, turbulence forcing is necessary. The forcing magnitude is increased $0.5 \mathrm{~L}$ downstream of the inlet to reach the desired TKE value. The velocity field forcing is stopped after a distance of $8 L$ and the velocity fluctuations decay rapidly, such that there are no negative axial velocities at the outlet. The forcing method used is briefly described in subsection 2.4. For each simulation, the mean inlet velocity is constant throughout the simulation and selected to match the turbulent flame speed of that simulation. In practice, the flames drift slightly, but the drift is negligible compared to the domain length. The simulations are performed for at least 50 eddy turnover times, $\tau$, and statistics are computed using the last $30 \tau$ to remove any initial transient effects. All simulations are performed on uniform grids with constant $\Delta x$ spacing in all three directions and offer sufficient resolution of the small-scale turbulence $(\Delta x \leq 2 \eta)$ [23] and sufficient resolution of the flame $\left(\Delta x \leq l_{F} / 20\right)$ [24]. Grid resolution is discussed further in A.

[Fig. 1 about here.] 


\subsection{Simulation parameters}

The unburnt gas is a lean $n-\mathrm{C}_{7} \mathrm{H}_{16} /$ air mixture at standard pressure $\left(P_{0}=1 \mathrm{~atm}\right)$ and varying temperatures. The equivalence ratio $(\phi=0.9)$ is the same for all simulations and air is $23.2 \% \mathrm{O}_{2}$ and $76.8 \% \mathrm{~N}_{2}$ by mass. The unburnt temperatures $\left(T_{u}\right)$ and turbulent intensities $\left(u^{\prime}\right)$ are changed to cover a range of reaction zone Karlovitz numbers clustered around the thin to broken/distributed reaction zones regimes. Figure 2 shows where the simulations stand on a typical regime diagram, and Table 1 summarizes the simulation parameters. For most cases, two simulations are performed: one with non-unity Lewis numbers and one with unity Lewis numbers (subscript 1). Simulations $A$ and $A_{1}$ are expected to be representative of the thin reaction zones regime. Simulations $B$ and $B_{1}$ were previously reported in Savard et al. [20] and were selected to fall close to the transition from thin to broken/distributed reaction zones. They have the same unburnt temperature as case A with an increased turbulence intensity. The turbulence intensity ratio $\left(u^{\prime} / S_{L}\right)$ and length scale ratio $\left(l / l_{F}\right)$ of simulations $\mathrm{C}$ are held approximately the same as for case B, while the unburnt temperature is increased. The unburnt Karlovitz numbers (Ka) are thus similar, but the reaction zone Karlovitz numbers $\left(\mathrm{Ka}_{\delta}\right)$ are different. Finally, simulations $\mathrm{D}$ and $\mathrm{D}_{1}$ use the same unburnt temperature as $\mathrm{C}$ with a further increase in turbulence intensity, increasing further the Karlovitz numbers. Those simulations are expected to be representative of the distributed burning regime.

Simulation $C^{\prime}$ uses an intermediate unburnt temperature of $500 \mathrm{~K}$ with a turbulence intensity ratio resulting in the same reaction zone Karlovitz number as case $\mathrm{C}$ (albeit at an higher unburnt Karlovitz number). This simulation is used to highlight the importance of using the reaction zone Karlovitz number (with the Kolmogorov length scale at the reaction zone) to investigate the influence of turbulence on the 
chemical sources terms in simulations at different unburnt temperatures.

The objective of the present study is to investigate the effects of small-scale turbulence on the flame chemistry at high Karlovitz numbers. Given that purpose and computational cost considerations, a small integral length scale (roughly equal to the laminar flame thickness) is used. It is important to note that the domain width imposes a length scale and the simulations may be missing large scale effects. However, the focus of the paper is on the reaction zone, whose thickness is significantly smaller than the domain size $\left(L \sim 15 \delta_{F}\right)$. This way, the effects of small-scale turbulence on the flame can be isolated form large-scale effects (not specific to high Karlovitz number flames and already present in low Karlovitz number flames).

[Table 1 about here.]

[Fig. 2 about here.]

\subsection{Governing equations}

The low Mach number Navier-Stokes equations are solved

$$
\begin{gathered}
\frac{\partial \rho}{\partial t}+\nabla \cdot(\rho \mathbf{u})=0 \\
\frac{\partial \rho \mathbf{u}}{\partial t}+\nabla \cdot(\rho \mathbf{u u})=-\nabla p+\nabla \cdot \tau+\mathbf{f}, \\
\frac{\partial \rho Y_{i}}{\partial t}+\nabla \cdot\left(\rho \mathbf{u} Y_{i}\right)=-\nabla \cdot \dot{\mathbf{j}}_{\mathbf{i}}+\rho \dot{\omega}_{i}, \\
\frac{\partial \rho T}{\partial t}+\nabla \cdot(\rho \mathbf{u} T)=\nabla \cdot(\rho \alpha \nabla T)+\rho c_{p} \dot{\omega}_{T} \\
-\frac{1}{c_{p}} \sum_{i} c_{p, i} \mathbf{j}_{\mathbf{i}} \cdot \nabla T+\frac{\rho \alpha}{c_{p}} \nabla c_{p} \cdot \nabla T .
\end{gathered}
$$


In those equations, $\mathbf{u}$ is the velocity vector, $\rho$ is the fluid density, $\tau$ is the viscous stress tensor, $\mathbf{f}$ is the turbulence forcing term, $Y_{i}$ is the species mass fraction, $\dot{\omega}_{i}$ is the species production rate, $\mathbf{j}_{\mathbf{i}}$ is the species diffusion mass flux, $T$ is the temperature, $c_{p, i}$ is the species heat capacity, $c_{p}$ is the mixture heat capacity, $\alpha=\lambda /\left(\rho c_{p}\right)$ is the mixture thermal diffusivity, $\lambda$ is the mixture thermal conductivity, and $\dot{\omega}_{T}=-\frac{1}{c_{p}} \sum h_{i} \dot{\omega}_{i}$ is the temperature production term (heat release). The species diffusion term is defined as:

$$
\mathbf{j}_{\mathbf{i}}=-\rho D_{i} \frac{Y_{i}}{X_{i}} \nabla X_{i}-\rho Y_{i} \mathbf{u}_{\mathbf{c}}
$$

where $\mathbf{u}_{\mathbf{c}}$ is the correction velocity to ensure conservation of mass:

$$
\mathbf{u}_{\mathbf{c}}=-\sum_{i} \rho D_{i} \frac{Y_{i}}{X_{i}} \nabla X_{i}
$$

The fluid is treated as a perfect gas with the equation of state

$$
P_{0}=\rho R T \sum_{i} \frac{Y_{i}}{W_{i}}
$$

where $P_{0}$ is the thermodynamic pressure, $R$ is the universal gas constant, and $W_{i}$ is the species molecular weight. It should be noted that there is no viscous heating term in the temperature equation. This is equivalent to the addition of a cooling term to the internal energy equation and it ensures that the internal energy (and the temperature) can reach a stationary state [25].

The species viscosities $\mu_{i}$ are computed from standard gas kinetic theory [26] and the mixture-averaged viscosity is calculated using a modified form of Wilke's formula [27] (see B). The species thermal conductivities, $\lambda_{i}$, are computed using Eucken's formula [28] and the mixture-averaged thermal conductivity $\lambda$ is obtained 
following Mathur et al. [29]. The species diffusivities are computed as $D_{i}=\alpha / L e_{i}$, with the Lewis numbers $L e_{i}$ assumed to be constant throughout the flame. All nine simulations use the same Lewis numbers as the increase in unburnt temperature does not significantly change the Lewis numbers. The species Lewis numbers are taken from the simulation of a one-dimensional, laminar, premixed flames using full transport properties computed with FlameMaster [30]. They are listed in Table 2. Soret and Dufour effects were neglected. It was shown by Savard and Blanquart [21] that, in the one-dimensional laminar heptane flame considered, differences in species mass fractions and chemical source terms were negligible between mixture-averaged formulation for transport properties (including Soret effects) and constant Lewis numbers. The impact of these limitations in 3D turbulent flames is still unknown and is beyond the scope of the present work.

[Table 2 about here.]

The present study uses a reduced $n$-heptane chemical mechanism. The mechanism developed in Bisetti et al. [31] was further reduced from 47 species and 290 reactions to 35 species and 217 reactions. Species produced under rich conditions (mostly aromatic species) were removed. The mechanism used in this study does not contain low temperature chemistry (such as the formation of peroxide species $\mathrm{RO}_{2}$ ). While low temperature chemistry is known to be relevant to ignition studies, it is not expected to play a significant role in the present study on turbulent premixed flames.

The governing equations 3 to 9 are solved using the energy conservative, finite difference code NGA designed for the simulation of variable density, low Mach number, turbulent flows [32]. The simulations use second-order accurate spatial and temporal discretizations. A semi-implicit Crank-Nicolson time integration is 
used [33]. A preconditioning strategy based on a diagonal approximation of the chemical Jacobian [34] is used to integrate the chemical source terms. The diagonal elements are approximated as the inverse of the chemical consumption timescales. The preconditioning is applied within an iterative procedure for each time step, allowing suitable reduction of the residuals in unsteady reacting flows. The numerical integration method has been thoroughly valided in $0 \mathrm{D}, 1 \mathrm{D}$, and $3 \mathrm{D}$ cases (including the present turbulent flames). The third-order Bounded QUICK scheme, BQUICK [35], is used as the transport scheme of the species and temperature to ensure that the scalars remain within their physical bounds.

\subsection{Turbulence forcing}

The canonical flow configuration used in the present study lacks generation of turbulence due to large scale flow straining. Consequently, the turbulence is expected to decay. In the present simulations, the characteristic time scale for the decay of turbulent kinetic energy is smaller than the laminar flame time scale. The ratio of these two is in fact the inverse of the Karlovitz number. Velocity field forcing is thus necessary to maintain a constant turbulence intensity and achieve a statisticallystationary state.

Linear velocity forcing [36,37] was chosen for its physical nature and good stability properties. Linearly forced turbulent fields under comparable Reynolds numbers were analyzed in Ref. [37] and it was shown that the second- and third-order structure functions and the energy spectrum are self-consistent and in agreement with experimentally obtained data [38] of decaying grid turbulence. One advantage of the present linear forcing method is that an isotropic field is not imposed via the forcing and any physically-relevant anisotropy generated by the flame will be pre- 
served. The forcing method is implemented through the addition of a source term to the momentum equation

$$
\mathbf{f}(x, y, z, t)=\frac{\epsilon_{0}}{2 k(x, t)}(\rho \mathbf{u}(x, y, z, t)-\overline{\rho \mathbf{u}}(x, t))
$$

where $\epsilon_{0}$ is the desired dissipation rate and $k$ is the planar Favre-averaged TKE

$$
k=\frac{1}{2}\left(\widetilde{u^{\prime \prime 2}}+\widetilde{v^{\prime \prime 2}}+\widetilde{w^{\prime \prime 2}}\right)
$$

Planar Favre averages are defined as

$$
\widetilde{\xi}=\frac{\overline{\rho \xi}}{\bar{\rho}}
$$

with the standard (Reynolds) planar average

$$
\bar{\xi}(x, t)=\frac{1}{L^{2}} \int_{0}^{L} \int_{0}^{L} \xi(x, y, z, t) \mathrm{d} y \mathrm{~d} z
$$

As demonstrated in previous studies, the present forcing technique imposes a fixed integral length of approximately $1 / 5$ of the domain width and a constant dissipation rate throughout the flame [21,36,37]. Hence, the turbulent rms velocity ( $\left.u^{\prime}=\left(\epsilon_{0} l\right)^{1 / 3}\right)$ is fixed by $l$ and $\epsilon_{0}$. Under theses conditions, the turbulent kinetic energy was found to be almost constant [21].

Figure 3 shows the energy and dissipation spectra taken at a fixed plane in the unburnt gases and averaged over time. These correspond to two-dimensional three components velocity spectra computed in a $y$ - $z$ plane. When normalized by their respective Kolmogorov scales, all spectra collapse to a single curve. This confirms that, even in the absence of an inertial subrange in the present low Reynolds number 
simulations, the dissipation scales in the incoming turbulence are universal. Since the turbulence at the scale of the reaction zone is in the viscous dissipation range, the small scales interacting with the flame are expected to be the same whether the Reynolds number is high or low. Nevertheless, assessing Reynolds number effects in high Karlovitz number flames should be the subject of future work.

[Fig. 3 about here.]

\section{Results overview}

This section presents a qualitative overview of the simulations performed. The differential diffusion effects on the species mass fractions dependence on temperature are highlighted, and the influence of turbulence on the main chemical reactions contributing to fuel consumption and heat release rates is investigated.

\subsection{Global properties}

Figure 4 shows two-dimensional slices of vorticity magnitude and temperature for simulations A, B, C, and D using non-unity Lewis numbers (results of simulation $C^{\prime}$ are very similar to $C$ and will be discussed in Section 7). The slices were taken at arbitrary spatial and temporal locations (after the initial transient) and are representative of each simulation.

[Fig. 4 about here.]

Increasingly smaller scales are observed from case A to D. This is due to the decreasing Kolmogorov length scale. In all cases, smaller turbulent structures are observed in the preheat zone than in the reaction zone and in the burnt gases. As 
viscosity increases through the flame, the Kolmogorov length scale (defined as $\left.\eta=\left(\nu^{3} / \epsilon\right)^{1 / 4}\right)$ increases too. The vorticity contours also highlight the fact that, as the reaction zone Karlovitz number is increased, small-scale turbulent structures become more prominent in the burnt gases. In comparison to the respective incoming turbulence, smaller structures are observed in the burnt gases in cases $\mathrm{C}$ and $\mathrm{D}$ than cases $\mathrm{A}$ and $\mathrm{B}$. This is again an effect of the different viscosity ratios across the flame due to the change in unburnt temperature. Finally, the yellow and red lines indicate isocontours of temperature at $300 \mathrm{~K}$ below and above the corresponding temperature of peak fuel consumption rate in the turbulent flame. They illustrate the broadening of the reaction zone with increased Karlovitz number. The distributed nature of the reaction zone will be further analyzed in Section 5.

The temperature contours indicate that, in all cases, the flame appears thicker than the laminar flame but to a different extent. In case A, there is only a slight broadening of the preheat zone. The flame resembles a locally one-dimensional laminar flame. Signs of increased turbulent mixing are noticeable in cases B and C. The flame surface appears more wrinkled and the preheat zone is further broadened. Case D presents more small-scale turbulent structures and enhanced turbulent mixing.

Table 3 summarizes the average turbulent flame speed $\left(S_{T}\right)$, its standard deviation, and the fuel effective reaction zone thickness, all normalized by their respective laminar flame values. The turbulent flame speed is defined here as:

$$
S_{T}=\frac{1}{\left(\rho Y_{F}\right)_{u} L^{2}} \int_{V} \rho \dot{\omega}_{F} \mathrm{~d} V
$$

The effective reaction zone thickness (equivalent to the turbulent flame brush thickness of the whole flame) is defined here as the volume where the fuel burning rate 
is greater than $5 \%$ of the laminar value divided by the cross-sectional area of the $y-z$ plane,

$$
\delta_{T}=\frac{\operatorname{Vol}\left(\dot{\omega}_{F}>0.05 \dot{\omega}_{F, \mathrm{lam}}\right)}{L^{2}} .
$$

As expected, the turbulent flame speed increases with $\mathrm{Ka}_{\delta}$ and significant fluctuations are present. The effective reaction zone thickness also increases with $\mathrm{Ka}_{\delta}$, showcasing the broadening and increase in surface area of the flame with increased turbulence intensity. Note that the reaction zone can still be thin despite $\delta_{T}>1$. The effective reaction zone thickness is affected by the increase in surface area and thus does not represent the local reaction zone thickness. There is also a clear difference between non-unity Lewis number and unity Lewis number simulations. The ratio of turbulent to laminar flame speed is always lower when differential diffusion is included, although the difference is reduced as $\mathrm{Ka}_{\delta}$ is increased.

\section{[Table 3 about here.]}

\subsection{Turbulent flame structure}

Following the qualitative overview and global quantities presented in Section 3.1, the local flame structure is now investigated. As mentioned earlier, cases B and $\mathrm{B}_{1}$ have already been investigated by Savard et al. [20]. They reported that, in case $\mathrm{B}_{1}$ with unity Lewis numbers, the conditional mean profiles of species mass fractions with respect to temperature followed very closely the profiles of a one-dimensional, unstretched laminar flame at the same conditions. In contrast, in case B, the turbulent flame structure lied between that of a full transport and a unity Lewis number flamelet. This is a consequence of the increased effective species diffusivity due to turbulent mixing $[2,22]$. It is thus expected that, as the turbulence intensity is increased, differential diffusion effects would be reduced. This is verified in the 
present study by examining the conditional mean with respect to temperature of two representative species.

Figures 5 and 6 present conditional means of $n-\mathrm{C}_{7} \mathrm{H}_{16}$ and $\mathrm{C}_{2} \mathrm{H}_{4}$ mass fractions from simulations $\mathrm{A}$ (lowest $\mathrm{Ka}_{\delta}$ ) through $\mathrm{D}$ (highest $\mathrm{Ka}_{\delta}$ ). In all unity Lewis number cases (subscript 1), the conditional mean of the fuel mass fractions follows closely the unity Lewis laminar flame. The fuel mass fraction exhibits small differences between cases $\mathrm{A}$ and $\mathrm{A}_{1}$ and $\mathrm{B}$ and $\mathrm{B}_{1}$. No noticeable differences are observed between cases $\mathrm{C}$ and $\mathrm{C}_{1}$ and $\mathrm{D}$ and $\mathrm{D}_{1}$. The same trends are observed for the mass fraction of $\mathrm{C}_{2} \mathrm{H}_{4}$, a key chemical intermediate in the cracking of the fuel, shown in Fig. 6.

For both species, the profiles from the non-unity Lewis number turbulent simulations are closer to the unity Lewis number laminar flame than the non-unity Lewis number laminar flame. It should be noted that case A presents the largest departure from the unity Lewis number 1D profiles and that departure is reduced as the turbulence level is increased. This is consistent with the reduction of differential diffusion effects with increased turbulent mixing [22]. While differential diffusion effects appear negligible for flames $\mathrm{C}$ and $\mathrm{D}$, it still plays an important role on chemical source terms (as will be shown in Section 4).

Finally, one might expect that fuel cracking (i.e. fuel being consumed at lower temperatures than in the laminar flame) may occur in turbulent flames due to a thicker flame and thus longer residence time. Such early fuel cracking would result in a mean fuel mass fraction profile below that of the corresponding laminar flame. This was not observed here despite the high unburnt temperature of $800 \mathrm{~K}$.

[Fig. 5 about here.] 
[Fig. 6 about here.]

\subsection{Chemical pathways}

Since the present study is focused on the effects of turbulence on the fuel consumption and heat release rates, it is of interest to examine if the chemical pathways are affected by changes in the turbulence intensity. In this subsection, the main contributing reactions to fuel consumption and heat release rates are compared between the laminar and turbulent simulations.

In the laminar simulations, the following three reactions account for more than $95 \%$ of the overall fuel consumption rate

$$
\begin{gathered}
n-\mathrm{C}_{7} \mathrm{H}_{16}+\mathrm{H} \rightarrow 2-\mathrm{C}_{7} \mathrm{H}_{15}+\mathrm{H}_{2}, \\
n-\mathrm{C}_{7} \mathrm{H}_{16}+\mathrm{OH} \rightarrow 2-\mathrm{C}_{7} \mathrm{H}_{15}+\mathrm{H}_{2} \mathrm{O}, \\
n-\mathrm{C}_{7} \mathrm{H}_{16}+\mathrm{O} \rightarrow 2-\mathrm{C}_{7} \mathrm{H}_{15}+\mathrm{OH} .
\end{gathered}
$$

Table 4 lists the contributions (in percentage) of the three reactions to the overall fuel consumption rate of the corresponding flame. The contribution of the different reactions is very similar between laminar flames and turbulent flames. Cases A and $\mathrm{D}$ are selected as they have the lowest and highest reaction zone Karlovitz number, respectively. The percentage of contribution of each reaction are essentially unchanged from laminar to highly turbulent flames, with and without differential diffusion. In other words, turbulence and differential diffusion effects do not change the balance between the different fuel consumption reactions.

[Table 4 about here.] 
Considering the present mechanism, a large number of reactions present non-negligible heat release rates (above $5 \%$ of the total). Among them, the following two reactions contribute significantly to the overall heat release rate.

$$
\begin{gathered}
\mathrm{CH}_{3}+\mathrm{O} \rightarrow \mathrm{CH}_{2} \mathrm{O}+\mathrm{H}, \\
\mathrm{HCO}+\mathrm{H} \rightarrow \mathrm{CO}+\mathrm{H}_{2} .
\end{gathered}
$$

The contributions to the overall heat release rate from these two reactions are shown for the different laminar flames and turbulent cases A and D in Table 4. The heat release rate of each reaction is normalized by the peak overall heat release rate of the corresponding flame. For both reactions, the contribution to the total heat release rate remains largely unchanged between the laminar and turbulent cases. The fuel consumption reactions are not among the main contributors to heat release. This is because $n$-heptane breaks down into small $\mathrm{C}_{2}$ and $\mathrm{C}_{3}$ fragments (mostly $\mathrm{C}_{2} \mathrm{H}_{4}$ and $\mathrm{C}_{3} \mathrm{H}_{6}$ ) before the heat release layer.

In summary, for both fuel consumption and heat release rates, there is no significant effect of turbulence intensity on the main contributing reactions. The important reactions in the laminar flames remain the most important in the turbulent flames and their relative contributions are largely unchanged. Differences in absolute magnitudes will be discussed in the following sections.

\section{Differential diffusion effects}

In this section, the effects of differential diffusion on the fuel consumption and heat release rates are investigated. 
Contour plots of the fuel consumption rate for the eight different cases are presented in Fig. 7. A white line is super-imposed to show the location of the peak fuel consumption rate. It is defined as the isocontour $T=T_{\text {peak }}$, the temperature of peak source term in the corresponding turbulent flame. In cases A and B, the reaction zone remains thin but signs of local extinctions are observed for the nonunity Lewis number simulations. This was already observed by Savard et al. [20]. The Kolmogorov length scale in the unburnt gas is smaller than the laminar reaction zone thickness in all cases (A through D). However, it increases significantly through the flame due to the large increase in kinematic viscosity. In fact, for cases $\mathrm{A}$ and $\mathrm{B}$, the Kolmogorov length scale at the reaction zone $\left(\eta_{\delta}=1.2 \times 10^{-4}\right.$ and $\left.7 \times 10^{-5}\right)$, evaluated as $\eta_{\delta}=\left(\nu_{\delta}^{3} / \epsilon\right)^{1 / 4}$, is not significantly smaller than the laminar reaction zone thickness $\left(\delta_{F, \text { lam }}=1.5 \times 10^{-4}\right)$. As a result, turbulence does not significantly broaden the reaction zone.

As mentioned previously, there is a distinct change from case B to case $\mathrm{C}$. The Karlovitz number in the unburnt gases is the same in cases $\mathrm{B}$ and $\mathrm{C}$ but noticeable differences are observed since the Karlovitz number at the reaction zone is larger in case $\mathrm{C} . \mathrm{As}_{\mathrm{Ka}}$ is increased, the source terms exhibit larger fluctuations and local broadening of the reaction zone is observed. Finally, the fuel consumption rate contours of case D appear to be more diffused as chemical reactions occur further away from the iso-surface. This is characteristic of distributed burning.

[Fig. 7 about here.]

Figures 8 and 9 present conditional means in temperature space of the normalized fuel consumption and heat release rates for the different non-unity Lewis number cases. Only the mean source terms are shown but it should be noted that, as turbulence intensity at the reaction is increased, the fluctuations of the fuel con- 
sumption rate are enhanced significantly. A characterization and quantification of these fluctuations will be provided later in Section 6. For both fuel and heat release, simulations including differential diffusion exhibit a lower mean source term than the corresponding laminar flame with differential diffusion, but the conditional mean approaches that of the laminar flame without differential diffusion as $\mathrm{Ka}_{\delta}$ is increased. It is interesting to evaluate quantitatively these effects with increasing $\mathrm{Ka}_{\delta}$.

Figure 10a shows the shift in temperature of the peak fuel consumption rate for the non-unity and unity Lewis number turbulent flames. The peak temperature corresponds to the location of the maximum of the conditional means, shown in Fig. 8. Note that the peak temperature of heat release rate is not shown as it does not change significantly between non-unity and unity laminar and turbulent flames. For cases A and B, the peak fuel source term in the turbulent flame occurs at the same temperature as the 1D laminar flame with differential diffusion. In case C, the peak fuel consumption rate is observed at a slightly higher temperature than in the laminar non-unity Lewis number flame. This effect is more pronounced in case $\mathrm{D}$ as the temperature of peak source term approaches that of the laminar unity Lewis number flame. This is one of many indications that, as turbulent mixing is enhanced, differential diffusion effects are reduced. It should be noted that, while the dependence of species mass fractions on temperature showed relatively small Lewis number effects even in case A (Section 3.2), differential diffusion effects on the chemical source terms are more pronounced and still present at high Karlovitz numbers.

The change in peak temperature can be explained by a change in the effective Lewis numbers due to enhanced turbulent mixing. To quantify this change, the 
semi-empirical model of Savard and Blanquart [22] is considered

$$
L e_{i, \mathrm{eff}}=\frac{1+a_{1} \mathrm{Ka}_{\delta}}{\frac{1}{L e_{i}}+a_{1} \mathrm{Ka}_{\delta}}
$$

As the focus is placed on the reaction zone and not the preheat zone, the unburnt Karlovitz number $\mathrm{Ka}$ was replaced by $\mathrm{Ka}_{\delta}$. Similarly to [22], the proportionality coefficient is adjusted to best fit the data $\left(a_{1}=0.01\right)$. One-dimensional laminar flames are computed with these effective Lewis numbers and the predictions of the peak temperature of the fuel consumption rate are shown in Fig. 10a. The change in peak temperature of the laminar flames at both $298 \mathrm{~K}$ and $800 \mathrm{~K}$ are similar and thus a single curve is shown for clarity. A good agreement between the model and the turbulent flames simulations is found.

[Fig. 8 about here.]

[Fig. 9 about here.]

Figure $10 \mathrm{~b}$ presents the maximum values of the conditional means of $\dot{\omega}_{F}$ and $\dot{\omega}_{T}$ as a function of the reaction zone Karlovitz numbers for the different simulations using both non-unity and unity Lewis numbers. The conditional means were computed using at least 30 data files (to ensure converged statistics) and the standard deviations of the means are negligible. There are, however, large fluctuations of the chemical source terms around the mean values, as will be discussed in Section 6. The mean fuel consumption rates of the unity Lewis number cases are all close to the laminar values. While this had been observed in previous studies [20,21] for the fuel consumption in case $B_{1}$, it is of interest to note that there is no Karlovitz number effect on the mean fuel consumption and heat release rates in the unity Lewis number cases. On the other hand, the mean fuel consumption rate is smaller than 
that of the corresponding laminar flame in all non-unity Lewis number cases. As $\mathrm{Ka}_{\delta}$ is increased, the conditional means of the non-unity flames get closer to those of the corresponding unity Lewis number flame. It appears that, in the limit of high Karlovitz numbers, the fuel consumption rate approaches the laminar value without differential diffusion. Once again, this is consistent with previous observations that differential diffusion effects appear to weaken as turbulence intensity increases. In previous studies of distributed flames [11,14], it was observed that the fuel consumption rate was enhanced in low Lewis number hydrogen flames and remained close to the laminar values in methane and propane flames. This is consistent with the present observations.

The lower mean fuel consumption and heat release rates in the present non-unity Lewis number simulations can be attributed to curvature effects. It was shown by Savard and Blanquart [21], in case B, that low fuel consumption rates are correlated with regions of high curvature, suggesting the presence of focusing/defocusing effects. As differential diffusion effects are diminished, the chemical source terms are less sensitive to curvature. This difference in fuel consumption rates can explain the differences in the flame speeds reported in Table 3. As discussed in Savard and Blanquart [21], the ratio of turbulent to laminar flame speeds $\left(S_{T} / S_{L}\right)$ can be explained by the turbulent flame surface area $\left(A_{T} / A\right.$, where $A$ is the cross-sectional area) and the mean turbulent to laminar chemical source term ratio $\dot{\omega} / \dot{\omega}_{\text {lam. }}$. For the unity Lewis number simulations, $\mathrm{A}_{1}$ through $\mathrm{D}_{1}$, since the source term ratio is unity, the change in flame speeds is due to an increase in flame surface area $A_{T} / A$ with increasing $\mathrm{Ka}_{\delta}$. Finer turbulent structures within the flame (due to the larger $\mathrm{Ka}_{\delta}$ ) lead to an enhanced area. The difference between the non-unity and unity Lewis number simulations at the same $\mathrm{Ka}_{\delta}$ are due to the difference in $\dot{\omega} / \dot{\omega}_{\text {lam }}$ since, for a given $\mathrm{Ka}_{\delta}$, the flame surface area is roughly constant. Thus, the increase in $S_{T} / S_{L}$ 
with $\mathrm{Ka}_{\delta}$ for the non-unity Lewis number cases (A through D) is due to both an increase in $\dot{\omega} / \dot{\omega}_{\text {lam }}$ and an increase in $A_{T} / A$. This is summarized in Table 5.

[Table 5 about here.]

Heat release rate shows similar trends as the fuel consumption rate, namely: the conditional means of unity Lewis number simulations are close to the corresponding laminar value; and simulations with differential diffusion show a lower mean but a noticeable increase in $\left\langle\dot{\omega}_{T}\right| T>$ with $\mathrm{Ka}_{\delta}$, getting closer to the unity Lewis number simulations. Note that the maximum values of the conditional means of heat release (Fig. 10b) are computed at the temperature of peak heat release rate of the corresponding flame. This temperature being larger than the temperature of peak fuel consumption rate, there is a reduction in the Kolmogorov length scale at the corresponding $T_{\text {peak }}$. Additionally, the width of the heat release layer is slightly larger than that of the fuel consumption layer. Hence, the reaction zone Karlovitz number for heat release differs from that of the fuel. That being said, the difference is small and does not change any of the trends presented here or in following sections. Thus, for simplicity, both fuel and heat release quantities are plotted against the fuel's $\mathrm{Ka}_{\delta}$.

[Fig. 10 about here.]

\section{Distributed burning}

In this section, the transition towards distributed burning is investigated. Figures 4 and 7 seem to indicate that a transition towards distributed burning is observed as $\mathrm{Ka}_{\delta}$ is increased. Distributed burning is characterized by a widening of the reaction zone, and is due to increased turbulent mixing in that region of the flame [11]. 
The first step is to quantify the widening of the reaction zone in physical space by evaluating the mean fuel consumption rate conditional to the normal distance to the isosurface of $T_{\text {peak }}$. This post-processing step is performed using a level set method [39]. A level set function is first created as $d(x, y, z)=T(x, y, z)-T_{\text {peak }}$. It is then reinitialized into a signed distance function using a Fast Marching Method (FMM) algorithm. The conditional mean of the burning rate as a function of distance from the peak temperature is shown in Figs. 11a and 11b. The distance has been normalized by the laminar reaction zone thickness, defined as the distance over which the fuel burning rate is above $5 \%$ of the peak laminar value. In cases $\mathrm{A}$ and $\mathrm{B}$, the fuel source term follows a similar profile as the laminar flame (albeit with a smaller magnitude). This is an evidence that cases $\mathrm{A}$ and $\mathrm{B}$ fall in the thin reaction zone regimes. A slight widening of the reaction zone is observed at small values of $\dot{\omega}_{F}$. In cases $\mathrm{C}$ and $\mathrm{D}$, this widening is more noticeable, and nonnegligible fuel consumption rates are observed at distances greater than the laminar reaction zone thickness. To quantify the widening of the reaction zone as the turbulence level is enhanced, Fig. 11c presents the percentage of the total burning rate occuring outside the laminar reaction zone thickness for the different cases

$$
\frac{\int_{|d|>\delta_{F, \operatorname{lam} / 2}} \dot{\omega}_{F} \mathrm{~d} V}{\int_{V} \dot{\omega}_{F} \mathrm{~d} V} .
$$

The percentage of reactions occuring outside of the laminar reaction zone thickness varies from $8 \%$ in cases A and B to $20 \%$ in case D. It is important to note that similar trends are observed in both non-unity and unity Lewis number simulations. In other words, the widening of the reaction zone is not an effect of differential diffusion.

[Fig. 11 about here.] 
The broadening of the reaction zone in physical space can have two different causes. First, in phase space, the chemical reactions may be occuring over a wider temperature range. Second, it could be the consequence of a change in local temperature gradients. Table 6 presents the average fuel reaction zone thickness in temperature space $\overline{\Delta T_{F}}$ and the average reaction zone thickness in physical space $\overline{\delta_{F}}$, both normalized by the corresponding laminar values. The thickness in temperature space corresponds to the temperature range where the mean fuel consumption rate (see Fig. 8) is above $5 \%$ of the laminar value. The physical thickness of the reaction zone corresponds to the local distance around the peak source term where its value is above $5 \%$ of the laminar value (see Fig. 11). The main observation is that the reaction zone is not wider than the laminar flame in temperature space. Consistently with the results of Section 3.3, reactions occur in the same temperature range as the laminar flames. Hence, the widening of the reaction zone is due to a change in the local temperature profile. To better illustrate this conclusion, Fig. 12 presents the conditional mean of temperature as a function of the distance from the peak temperature for the turbulent and laminar flame simulations. It is seen that, as the reaction zone Karlovitz number is increased, the temperature profile is flattened. Temperatures close to the peak temperature are observed over a larger distance away from the peak temperature.

[Table 6 about here.]

[Fig. 12 about here.]

The widening of the reaction zone can be explained (once again) by the enhanced turbulent diffusivity. First, let us assume that the reaction zone thickness scales as 
the square root of the product of the diffusivity and the chemical time scale

$$
\delta_{F} \propto \sqrt{D t_{c}}
$$

Consider again the semi-empirical model of Savard and Blanquart [22] for the turbulent diffusivity

$$
\frac{D_{T}}{D} \propto a_{2} \mathrm{Ka}_{\delta}
$$

Assuming that the chemical time scales are essentially unchanged between laminar and turbulent cases, a model for the turbulent reaction zone thickness follows

$$
\frac{\overline{\delta_{F}}}{\delta_{F, l a m}} \propto \sqrt{1+a_{2} \mathrm{Ka}_{\delta}} .
$$

The proportionality coefficient is adjusted to best fit the data $\left(a_{2}=0.02\right)$ and remains close to that obtained for Eq. (18) $\left(a_{1}=0.01\right)$. The functional form of the empirical models presented here should be applicable to other fuels and conditions. However, the exact numerical values of the proportionality coefficients might change. The prediction from the model is compared with the results in Fig. 13. A good agreement between the model and the turbulent flames simulations is found. This is the final evidence that the broadening of the reaction zone is due to reduced temperature gradients because of enhanced turbulent mixing. It should be noted that, in addition to enhanced turbulent diffusivity, increased occurence of high curvatures could lead to the observed widening of the reaction zone. It is, however, difficult to differentiate these effects.

[Fig. 13 about here.]

In previous studies $[11,14]$, distributed burning was related to an exponential distribution in the probability density function (pdf) of $|\nabla \rho|$, characteristic of turbulent 
scalar mixing. It was shown that in low Karlovitz number (non-distributed) flames, the pdf presented a more rapid decay than exponential for large gradients. An exponential decay of the pdf of $|\nabla \rho|$ is equivalent to a log-normal distribution for $\chi=2 \alpha|\nabla T|^{2}$. This is characteristic of turbulent mixing of a passive scalar [40-42]. While it is a consequence of turbulent eddies penetrating the reaction zone, it is not an evidence of distributed burning. Again, the widening of the reaction zone is due to smaller density/temperature gradients (Fig. 12).

Finally, it is important to note that despite the clear widening of the reaction zone most of the reactions still occur in a thin reaction zone. Additionally, the structure of the reaction zone above $\sim 20 \%$ of the peak laminar value is very similar to that of the laminar flame (Figs. 12a and 12b). These observations, combined with the previous observation of Savard and Blanquart [21] that the fuel consumption locally scales like its value at $T_{\text {peak }}$, justify considering a flame isosurface at the peak temperature as will be done in the following section.

\section{Local extinction}

This section focuses on the third and final objective, namely the occurence of local extinctions in the fuel consumption and heat release rates. Figure 14a presents standard deviations of the fuel consumption and heat release rate at the corresponding peak temperatures as a function of the reaction zone Karlovitz numbers. Both series of flames (unity and non-unity Lewis numbers) present similar fuel consumption rate standard deviations. The amplitudes of fluctuations increase from cases A to D, consistently with the increase in small-scale turbulence at the reaction zone. This is also in agreement with the fluctuations in turbulent flame speeds reported in Table 3. In all cases, the magnitude of the standard deviation is comparable to that 
of the mean. In the case of heat release, the standard deviations are slightly larger with differential diffusion effects and increase with higher reaction zone Karlovitz number in the unity Lewis number simulations. It is interesting to note that differential diffusion effects have a clear influence on the mean chemical source terms (Fig. 10b) but show a much smaller effect on the standard deviations. Chemical source term fluctuations are primarily caused by the intense turbulence and only show little impact on heat release rates.

[Fig. 14 about here.]

Figure $14 \mathrm{~b}$ presents the probability density of $\dot{\omega}_{F}$ (taken at the corresponding isosurface $T=T_{\text {peak }}$ ) for the different cases using non-unity Lewis numbers. All simulations exhibit smaller fuel consumption rates compared to the laminar value of 1. This is consistent with the mean burning rates reported earlier in Section 4 (Fig. 10b). As turbulence intensity is increased, the peak of the distribution is followed by a sharper decrease. The long tails (for large values) in cases C and D correspond to the increased fluctuations in burning rate mentioned earlier. The insert with a logarithmic scale illustrates the change in distribution with increased turbulence intensity. The pdfs of the burning rate are approaching a log-normal distribution in cases $\mathrm{C}$ and $\mathrm{D}$.

Figure $14 \mathrm{c}$ presents the probability density of $\dot{\omega}_{T}$ (taken at the corresponding $T_{\text {peak }}$ of heat release) for the different cases using non-unity Lewis numbers. Higher probability of small heat release rates are found at lower turbulence intensities. As $\mathrm{Ka}_{\delta}$ is increased, the peak of the pdf shifts towards larger values. This shift occurs to greater extent than for the fuel consumption rate. The shape of the pdf also changes significantly. Once again, case D is approaching a log-normal distribution. 
Local extinctions are defined here as the fuel consumption rate being smaller than $5 \%$ of the laminar value on the isosurface $T=T_{\text {peak }}[21,43]$. They are observed with similar probability in cases A (2\%) and B (3\%) and lower probability in cases $\mathrm{C}(<1 \%)$ and $\mathrm{D}(<0.1 \%)$ as shown in Fig. 15. This decrease in probability of extinction events with increasing $\mathrm{Ka}_{\delta}$ is interesting as it was shown in Fig. 14a that source term fluctuations increased with higher $\mathrm{Ka}_{\delta}$. The decrease in extinctions and increase in fluctuations can be attributed to the transition to a distributed burning regime. The reaction zone is uniformized by enhanced turbulent mixing, thus reducing the probability of local extinction. This also illustrates that extinction events are only present for the non-unity Lewis number cases, over the present range of Karlovitz numbers.

[Fig. 15 about here.]

These observations, at different Karlovitz numbers, are consistent with the previous observation [21] in case B that local extinctions (in non-unity Lewis number flames) are correlated with regions of high curvature (i.e. curvature of the size of the reaction zone thickness). Intense turbulence is required to produce such high curvatures in thermo-diffusively stable flames. More precisely, the fuel mass fraction is correlated with curvature [21], illustrating the defocusing/focusing effect observed in laminar and turbulent flames [44]. Extinction events are thus caused by a combination of intense turbulence and differential diffusion effects.

In summary, the probability of local extinction (or very low fuel consumption rate) increases as $\mathrm{Ka}_{\delta}$ is increased from a laminar flame to case $\mathrm{B}$ due to the larger curvatures. $\mathrm{As} \mathrm{Ka}_{\delta}$ is further increased the probability of extinction decreases. This is a consequence of the transition towards unity effective Lewis numbers. The gradual suppression of differential diffusion effects reduces the probability of extinction in 
regions of high curvature. In the present high Karlovitz number flames, no global extinction were observed and local extinctions were associated with differential diffusion effects. This is consistent with the observations of Aspden et al. [11,14].

\section{Karlovitz number definition}

It is common practice to classify turbulent premixed flames with respect to global, governing quantities evaluated in the unburnt gases, such as the unburnt Karlovitz number [2]. The simulations presented here highlight the fact that the Karlovitz number at the reaction zone (Eq. (2)) is the meaningful parameter to characterize the influence of small-scale turbulence on the chemical source terms. More precisely, simulations B and C are qualitatively and quantitatively different while having very similar values of the unburnt Karlovitz number $\mathrm{Ka}_{u}$ (220 and 204). On the other hand, simulations $\mathrm{C}$ and $\mathrm{C}^{\prime}$, with a similar $\mathrm{Ka}_{\delta}$ (73 and 70) but different $\mathrm{Ka}_{u}$ (204 and 648), have very similar average turbulent flame speeds as well as standard deviations (see Table 3). Additionally, their fuel consumption and heat release rates distributions, shown in Figs. 16a and 16b, are very close.

The reaction zone Karlovitz number is affected by the change in viscosity across the flame and does not take into account the density ratio. Under these conditions, the fact that simulations with the same reaction zone Karlovitz number but different density ratios ( $\mathrm{C}$ and $\left.\mathrm{C}^{\prime}\right)$ present very similar reaction zone characteristics is an illustration that the change in viscosity has a much greater impact on the small scales than the change in density. The dependence on the reaction zone Karlovitz number may have been expected, but it emphasizes that care has to be exercised when comparing simulations performed at the same $u^{\prime} / S_{L}$ and $l / l_{F}$ but using dif-

ferent unburnt temperatures. While $\mathrm{Ka}_{u}$ is appropriate to quantify the influence of 
turbulence on the preheat zone, $\mathrm{Ka}_{\delta}$ (with the Kolmogorov length scale evaluated at the reaction zone) should be used to describe the effect of the turbulence on the reaction zone.

In the present analysis, the reaction zone Karlovitz number is defined in terms of laminar flame properties and incoming turbulence quantities, similarly to the commonly used Karlovitz number evaluated in the unburnt gases. This is possible since $\epsilon$ is constant through the present flames (as a result of the forcing technique). However, this may not be the case in general and thus an accurate estimation of $\mathrm{Ka}_{\delta}$ would require knowledge of $\epsilon$ at the reaction zone.

[Fig. 16 about here.]

\section{Discussion and conclusion}

A series of direct numerical simulations of premixed $n$-heptane/air flames at different Karlovitz numbers have been performed using detailed chemistry. Different unburnt temperatures and turbulence intensities were used and their effects on the flame structure and chemical source terms were investigated. Differential diffusion effects have been systematically isolated by performing simulations with both nonunity and unity Lewis numbers. Three main observations were made.

First, the increase in turbulence intensity suppressed differential diffusion effects on the flame structure; the species mass fractions dependence on temperature was similar to that of a one-dimensional, unity Lewis number flame. However, differential diffusion effects on the chemical source terms were still present even at high Karlovitz numbers. The consequences were lower mean fuel consumption and heat release rates in the non-unity Lewis number simulations. As turbulence intensity at 
the reaction zone was increased (i.e. increase in $\mathrm{Ka}_{\delta}$ ), these rates are ultimately approaching the respective unity Lewis number values. This first set of observations has multiple implications for modeling. In the scope of LES, it indicates that mean quantities $\left(Y_{i}, \omega_{i}\right.$, etc) for unity Lewis number turbulent flames, such as methane flames, could be adequately modeled without detailed chemistry. Tabulated chemistry approaches based on laminar flames would be sufficient. However, this is not the case when significant differential diffusion effects are present, as for heavy hydrocarbon fuels. Thus, there is a need for reduced order models capable of predicting accurately the mean chemical source terms in non-unity Lewis number high Karlovitz flames.

Second, the transition to distributed burning was highlighted by measuring the width of the reaction zone in physical and temperature space. It was shown that the reaction zone's physical width increases with the reaction zone Karlovitz number. However, even at high Karlovitz numbers $\left(\mathrm{Ka}_{\delta} \sim 100\right)$, most of the reactions still occur in a thin reaction zone of a thickness comparable to that of a laminar flame.

Third, local extinctions and chemical source terms fluctuations were investigated and quantified through probability density functions at the temperature of peak reactions. Large fluctuations in fuel consumption rate were observed for both nonunity and unity Lewis number simulations, increasing with the reaction zone Karlovitz number. However, it is unclear, especially in the context of LES filtering, if these chemical source term fluctuations need to be incorporated in low-order models. These large fluctuations only lead to local extinction events in the non-unity Lewis number flames, and the probability of these events decreased at high Karlovitz numbers. In fact, less than $1 \%$ of the flame surface underwent extinction at $\mathrm{Ka}_{\delta}>10$. This was explained by the competing effects of increased curvatures and decreased 
differential diffusion effects as the reaction zone Karlovitz number was increased.

Throughout the analysis, it was shown that the reaction zone Karlovitz number (evaluated with the appropriate Kolmogorov length scale) was the relevant parameter to characterize the interaction of small-scale turbulence on the chemical source terms. Simulations with similar unburnt Karlovitz numbers but different reaction zone Karlovitz numbers showed distinct fuel consumption and heat release rates.

\section{A Grid resolution}

The grid spacing for all cases is chosen such that $\kappa_{\max } \eta>1.5$ everywhere in the domain, and is limited by the turbulence in the unburnt gases, where $\eta$ is the smallest. In order to verify the quality of the solution, case B with twice the number of grid points per direction was performed for 13 eddy turnover times (limited by the cost of such a simulation). Figure 17 compares the fuel burning rate statistics for the nominal $(1408 \times 128 \times 128)$ and refined $(2816 \times 256 \times 256)$ simulations. These differences are marginal for the purpose of the analysis made in this work. Since conditional means and standard deviations are moments of the probability density function, the fact that the pdfs are in excellent agreement indicates that the results would not change noticeably if the refined grid was used instead of the nominal grid. The conclusions are therefore grid independent.

[Fig. 17 about here.] 


\section{B Mixture-averaged viscosity}

In an effort to reduce computational cost, a simplified formula for the evaluation of the mixture-averaged viscosity is preferred over the commonly used Wilke's formula [27]. In the present work, the mixture-averaged viscosity is computed using a similar approach as the mixture-averaged thermal conductivity [29]

$$
\mu=\frac{1}{2}\left(\sum_{i=1} X_{i} \mu_{i}+\left[\sum_{i=1} \frac{X_{i}}{\mu_{i}}\right]^{-1}\right)
$$

where $X_{i}$ is the species mole fraction and $\mu_{i}$ is the species viscosity. This formulation is linear in the number of species rather than quadratic, which is the case of the common implementation of Wilke's formula.

The mixture-averaged viscosities computed using both Wilke's formula and Eq. (B.1) are compared in Fig. 18 in the case of the laminar $n$-heptane/air flame with $T_{u}=298 \mathrm{~K}$. The difference between the two methods is always less than $2 \%$. For the present 35 species mechanism, evaluation of the mixture-averaged viscosity is 70 times faster using Eq. (B.1).

[Fig. 18 about here.]

\section{Surface and volume weighting}

Different methods can be used to compute conditional means and conditional probability density functions, among which: surface area weighting and volume weighting. Consider, for example, the computation of conditional means $\langle y| x>$ or probability density functions $\mathrm{P}(y \mid x)$. Surface area weighting consists of generating an 
isosurface at the given value of $x$ and interpolating $y$ onto the isosurface. The mean or probability are then computed by weighting the values of $y$ by the corresponding face area. The creation of the isosurface and the interpolation often lead to an increase in computational cost. In contrast, in volume weighting, computational cells whose value of $x$ are within a small range (bin) of the desired value are considered. The mean or probability are then computed by weighting the values of $y$ by the corresponding cell volume. This method is simpler and computationally cheaper as it only requires binning the data. In the present study, surface area weighting is preferable in the context of model development. For example, a progress variable chemistry tabulation model requires the values of fluid properties and progress variable reaction rate on the progress variables isosurfaces.

The differences between the results obtained with each method are illustrated in Fig. 19, on the conditional means of fuel consumption rate for case C. In addition to surface area and volume weighting, a third conditional mean was computed using a volumetric (by bin) approach but weighting the values of $\dot{\omega}_{F}$ by the local value of $|\nabla T|$. In the present simulations, it is observed that, in comparison with surface area weighting, volume weighting decreases the relative importance of large fuel consumption rate regions while increasing that of small fuel consumption rate regions. This results in a significantly lower conditional mean around the peak temperature. At the high turbulence intensities considered here, large temperature gradients are observed in the reaction zone (at high fuel consumption rates). Due to those large gradients, the isosurfaces of temperature get closer together. Thus, there are more isosurfaces in a bin for large fuel consumption rates compared to small fuel consumption rates (small temperature gradients). This is the cause of the decreased weight of large fuel consumption rate regions with volume weighting.

In contrast, volume weighting using the norm of the gradient of temperature gives 
extremely similar result to surface area weighting. It is thus possible to obtain surface area weighted conditional means and probabilities without creating isosurfaces and interpolating 3D fields. This approach was used throughout this work.

[Fig. 19 about here.]

\section{Acknowledgments}

The authors gratefully acknowledge funding from Air Force Office of Scientific Research (FA9550-12-1-0472 and FA9550-12-1-0144) under supervision of Dr. Chiping Li and Fonds de Recherche du Québec - Nature et Techonologies for financial support. This research used resources of the National Energy Research Scientific Computing Center, a DOE Office of Science User Facility supported by the Office of Science of the U.S. Department of Energy under Contract No. DE-AC0205CH11231. This work used the Extreme Science and Engineering Discovery Environment (XSEDE), which is supported by National Science Foundation grant number ACI-1053575.

\section{References}

[1] N. Peters, The turbulent burning velocity for large-scale and small-scale turbulence, J. Fluid Mech. 384 (1999) $107-132$.

[2] N. Peters, Turbulent Combustion, Cambridge University Press, 2000.

[3] H. Pitsch, Large-eddy simulation of turbulent combustion, Ann. Rev. Fluid Mech. 38 (2006) $453-482$. 
[4] Y.-C. Chen, R. Bilger, Experimental investigation of three-dimensional flame-front structure in premixed turbulent combustion: Ii. lean hydrogen/air bunsen flames, Combust. Flame 138 (2004) 155 - 174.

[5] J. Hult, S. Gashi, N. Chakraborty, M. Klein, K. Jenkins, S. Cant, C. Kaminski, Measurement of flame surface density for turbulent premixed flames using plif and dns, Proc. Comb. Inst. 31 (2007) 1319 - 1326.

[6] M. Dunn, A. Masri, R. Bilger, A new piloted premixed jet burner to study strong finite-rate chemistry effects, Combust. Flame 151 (2007) 46 - 60.

[7] M. Dunn, A. Masri, R. Bilger, R. Barlow, G. Wang, The compositional structure of highly turbulent piloted premixed flames issuing into a hot coflow, Proc. Comb. Inst. 32 (2009) $1779-1786$.

[8] F. Carbone, J. Smolke, A. Fincham, F. Egolfopoulos, Characteristics of piloted premixed turbulent-jet flames of methane and $\mathrm{c}_{6}-\mathrm{c}_{8}$ hydrocarbons, in: Spring Technical Meeting of the WSSCI (2014), paper No. 14S-03, 2014.

[9] A. Aspden, J. Bell, M. Day, S. Woosley, M. Zingale, Turbulenceflame interactions in type ia supernovae, Astrophys. J. 689 (2008) 1173 - 1185.

[10] A. Aspden, J. Bell, S. Woosley, Distributed flames in type ia supernovae, Astrophys. J. 710 (2010) $1654-1663$.

[11] A. Aspden, M. Day, J. Bell, Turbulence-flame interactions in lean premixed hydrogen: transition to the distributed burning regime, J. Fluid Mech. 680 (2011) $287-320$.

[12] A. Aspden, M. Day, J. Bell, Turbulence-chemistry interaction in lean premixed hydrogen combustion, Proc. Comb. Inst.

[13] H. Wang, K. Luo, J. Fan, Direct numerical simulation and conditional statistics of hydrogen/air turbulent premixed flames, Energ. Fuel 27 (2013) 549 - 560. 
[14] A. Aspden, M. Day, J. Bell, Lewis number effects in distributed flames, Proc. Comb. Inst. 33 (2011) $1473-1480$.

[15] R. Sankaran, E. Hawkes, J. Chen, T. Lu, C. Law, Structure of a spatially developing turbulent lean methane-air bunsen flame, Proc. Comb. Inst. 31 (2007) 1291 - 1298.

[16] A. Poludnenko, E. Oran, The interaction of high-speed turbulence with flames: Global properties and internal flame structure, Combust. Flame 157 (2010) 995 - 1011.

[17] A. Poludnenko, E. Oran, The interaction of high-speed turbulence with flames: Turbulent flame speed, Combust. Flame 158 (2011) 301 - 326.

[18] H. Carlsson, R. Yu, X.-S. Bai, Flame structure analysis for categorization of lean premixed ch4/air and h2/air flames at high karlovitz numbers: Direct numerical simulation studies, Proc. Comb. Inst. 35 (2) (2015) 1425-1432.

[19] S. Won, B. Windom, B. Jiang, Y. Ju, The role of low temperature fuel chemistry on turbulent flame propagation, Combust. Flame 161 (2) (2014) 475 - 483.

[20] B. Savard, B. Bobbitt, G. Blanquart, Structure of a high karlovitz n-c7h16 premixed turbulent flame, Proc. Comb. Inst. 35 (2) (2015) 1377-1384.

[21] B. Savard, G. Blanquart, Broken reaction zone and differential diffusion effects in a high karlovitz n-c7h16 premixed turbulent flame, Combust. Flame.

[22] B. Savard, G. Blanquart, An a priori model for the effective species lewis numbers in premixed turbulent flames, Combust. Flame 161 (2014) 1547 - 1557.

[23] S. Pope, Turbulent Flows, Cambridge University Press, 2000.

[24] B. Bobbitt, G. Blanquart, Investigation of vortex-premixed flame interaction with detailed chemistry, in: Paper 070LT-0343, 8th US National Combustion Meeting, 2013.

[25] M. Petersen, D. Livescu, Forcing for statistically stationary compressible isotropic turbulence, Phys. Fluids 22 (2010) $1161011-11$. 
[26] O. Hirschfelder, C. Curtiss, R. Bird, Molecular Theory of Gases and Liquids, John Wiley and Sons, New York, 1954.

[27] C. Wilke, Journal of Chemical Physics 18 (4) (1950) 517-519.

[28] A. Eucken, Physik Z. 14 (1913) 324-333.

[29] S. Mathur, P. Tondon, S. Saxena, Thermal conductivity of binary, ternary and quaternary mixture of rare gases, Molecular Physics 12 (6) (1967) 569-579.

[30] H. Pitsch, $\quad$ FlameMaster: $\quad$ A computer program for 0D combustion and 1D laminar flame calculations. Available at http://www.itv.rwth-aachen.de/downloads/flamemaster/. (1998).

[31] F. Bisetti, G. Blanquart, M. Mueller, H. Pitsch, On the formation and early evolution of soot in turbulent nonpremixed flames, Combust. Flame 159 (2012) 317 - 335.

[32] O. Desjardins, G. Blanquart, G. Balarac, H. Pitsch, High order conservative finite difference scheme for variable density low mach number turbulent flows, J. Comp. Phys. 227 (15) (2008) $7125-7159$.

[33] C. Pierce, Progress-variable approach for large-eddy simulation of turbulent combustion, Ph.D. thesis, Stanford University (2001).

[34] B. Savard, Y. Xuan, B. Bobbitt, G. Blanquart, A computationally-efficient, semiimplicit, iterative method for the time-integration of reactive flows with stiff chemistry, J. Comp. Phys.

[35] M. Herrmann, G. Blanquart, V. Raman, Flux corrected finite volume scheme for preserving scalar boundedness in reacting large-eddy simulations, AIAA Journal 44 (2006) $2879-2886$.

[36] C. Rosales, C. Meneveau, Linear forcing in numerical simulations of isotropic turbulence: Physical space implementations and convergence properties, Phys. Fluids 17 (2005) $0951061-8$. 
[37] P. Carroll, G. Blanquart, The effect of velocity field forcing techniques on the nature and evolution of the karman-howarth equation, Journal of Turbulence 15 (2014) 429 448.

[38] L. Mydlarski, Z. Warhaft, On the onset of high-reynolds-number grid-generated wind tunnel turbulence, J. Fluid Mech. 320 (1996) 331 - 368.

[39] J. Sethian, Level Set Methods and Fast Marching Methods: Evolving Interfaces in Computational Geometry, Fluid Mechanics, Computer Vision and Materials Science, Cambridge University Press, 1999.

[40] E. Effelsberg, N. Peters, Scalar dissipation rates in turbulent jets and jet diffusion flames, Proc. Comb. Inst. 22 (1) (1989) 693-700.

[41] R. Antonia, K. Sreenivasan, Lognormality of temperature dissipation in a turbulent boundary layer, Phys. Fluids 20 (11) (1977) 1800-1804.

[42] C. Pantano, S. Sarkar, F. Williams, Mixing of a conserved scalar in a turbulent reacting shear layer, J. Fluid Mech. 481 (2003) 291-328.

[43] M. Day, J. Bell, P.-T. Bremer, V. Pascucci, V. Beckner, M. Lijewski, Turbulence effects on cellular burning structures in lean premixed hydrogen flames, Combust. Flame 156 (5) (2009) $1035-1045$.

[44] D. Haworth, T. Poinsot, Numerical simulations of lewis number effects in turbulent premixed flames, J. Fluid Mech. 244 (1992) 405-436. 


\section{List of Figures}

1 Schematic diagram of the flow configuration, from Savard et al. [20]. The domain is three-dimensional and periodic boundary conditions are used in the $y$ and $z$ directions.

2 Regime diagram showing current simulations.

3 Normalized energy and dissipation spectra for the different simulations. Two-dimensional three components spectra taken in a $y-z$ plane in the unburnt gases.

4 Two-dimensional slices of a $5 L \times L$ region centered around the flame showing magnitude of vorticity and temperature for the non-unity Lewis number cases, respectively. The yellow line indicates the $T_{\text {peak }}-300 \mathrm{~K}$ isocontour and the red line indicates the $T_{\text {peak }}+300 \mathrm{~K}$ isocontour. The vorticity ranges are satured at $[0,8 \mathrm{e} 4]$ $\left(s^{-1}\right),[0,1.6 \mathrm{e} 5]\left(s^{-1}\right),[0,1.6 \mathrm{e} 6]\left(s^{-1}\right)$, and $[0,8 \mathrm{e} 6]\left(s^{-1}\right)$. The temperature ranges are $[298,2200] \mathrm{K}$ for $\mathrm{A}$ and $\mathrm{B}$ and [800, 2400] $\mathrm{K}$ for $\mathrm{C}$ and $\mathrm{D}$.

5 Conditional means of fuel mass fractions.

6 Conditional mean of $\mathrm{C}_{2} \mathrm{H}_{4}$ mass fractions.

7 Two-dimensional slices of the fuel consumption rate (normalized by the peak value of the corresponding laminar flame). The fuel consumption rate range is satured at $[0,2]$ in each case. The isotherm of peak source term is also shown (white). The top figures correspond to the non-unity Lewis number simulations while the bottom figures correspond to the unity Lewis number cases.

8 Conditional means of normalized fuel consumption rate for non-unity Lewis number simulations. The fuel consumption rates are normalized by the peak value in the corresponding laminar flame.

9 Conditional means of normalized heat release rate for non-unity Lewis number simulations. The heat release rates are normalized by the peak value in the corresponding laminar flame. 
10 Temperature at which the peak burning rate is observed for all cases in (a). Conditional means of the normalized fuel consumption and heat release rates at the corresponding temperature of peak source term in (b). Values are normalized by the corresponding laminar flame.

11 Conditional mean of normalized fuel consumption rate as a function of distance from the peak temperature for the non-unity Lewis number simulations in (a) and (b) and fraction of the total burning rate occuring at $|d|>\delta_{F, \text { lam }} / 2$ for all cases in (c).

12 Conditional mean of temperature as a function of distance from the peak temperature for the non-unity Lewis number simulations.

13 Normalized fuel reaction zone thickness in physical space.

14 Standard deviations of the normalized fuel consumption and heat release rates in (a). Probability density function of the normalized fuel consumption rate in (b). Probability density function of the normalized heat release rate in (c). All taken at the corresponding peak temperature.

15 Probability of local extinction for the different cases.

16 Probability density function of the normalized fuel consumption and heat release rates at the corresponding $T_{\text {peak }}$.

17 Conditional mean and probability density function of the normalized fuel consumption rates. The probability density function was taken at the corresponding temperature of peak fuel consumption.

18 Mixture-averaged dynamic viscosity of laminar $n$-heptane/air flame computed using Wilke's formula and Eq. (B.1).

19 Conditional means of fuel consumption rate of case $\mathrm{C}$ calculated using volume weighting, surface area weighting, and weighting by the norm of the gradient of temperature. 


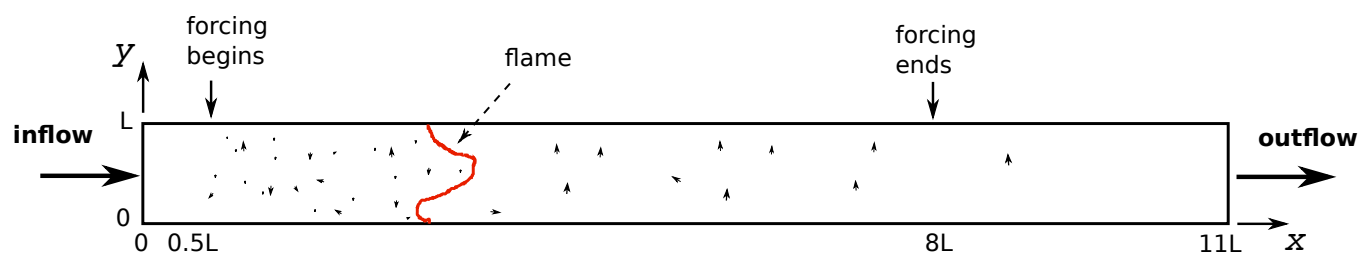

Fig. 1. Schematic diagram of the flow configuration, from Savard et al. [20]. The domain is three-dimensional and periodic boundary conditions are used in the $y$ and $z$ directions. 


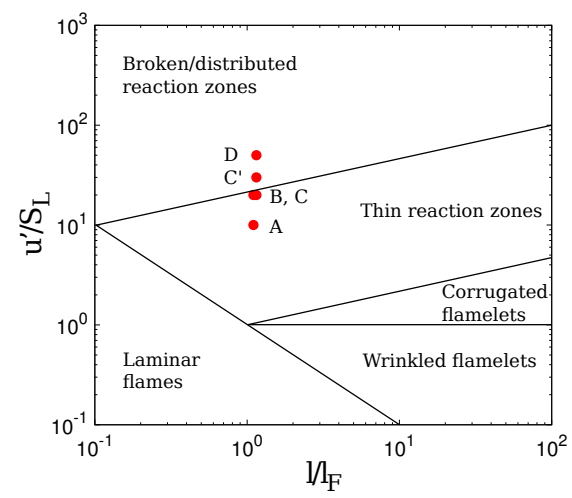

Fig. 2. Regime diagram showing current simulations. 


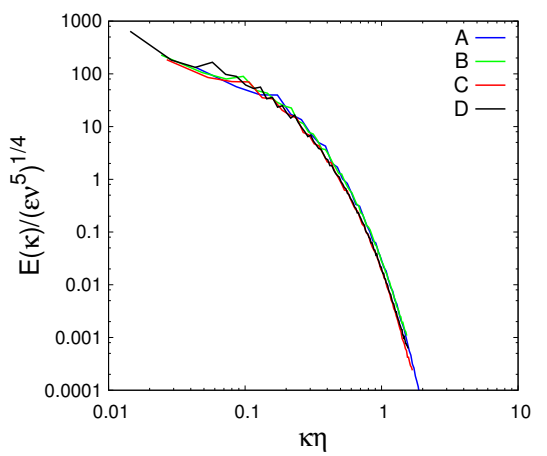

(a) Energy spectra

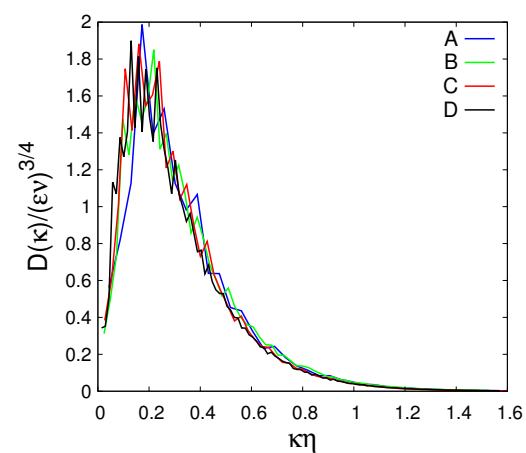

(b) Dissipation spectra

Fig. 3. Normalized energy and dissipation spectra for the different simulations. Two-dimensional three components spectra taken in a $y-z$ plane in the unburnt gases. 


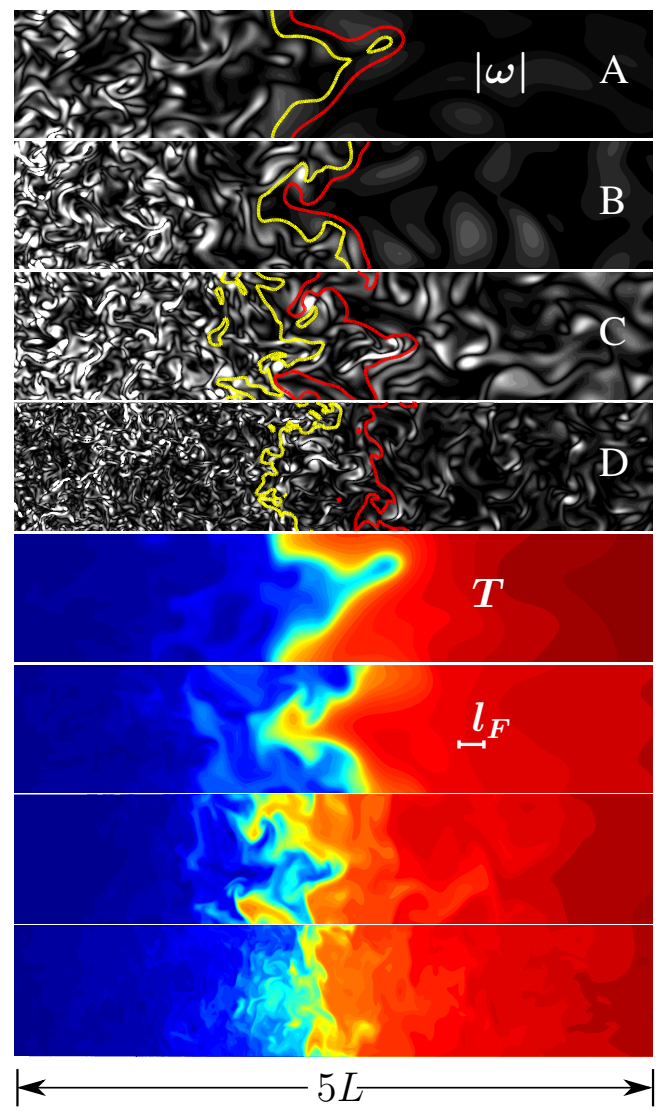

Fig. 4. Two-dimensional slices of a $5 L \times L$ region centered around the flame showing magnitude of vorticity and temperature for the non-unity Lewis number cases, respectively. The yellow line indicates the $T_{\text {peak }}-300 \mathrm{~K}$ isocontour and the red line indicates the $T_{\text {peak }}+300 \mathrm{~K}$ isocontour. The vorticity ranges are satured at $[0,8 \mathrm{e} 4]\left(s^{-1}\right),[0,1.6 \mathrm{e} 5]\left(s^{-1}\right),[0,1.6 \mathrm{e} 6]$ $\left(s^{-1}\right)$, and $[0,8 \mathrm{e} 6]\left(s^{-1}\right)$. The temperature ranges are $[298,2200] \mathrm{K}$ for $\mathrm{A}$ and $\mathrm{B}$ and $[800$, 2400] $\mathrm{K}$ for $\mathrm{C}$ and $\mathrm{D}$. 


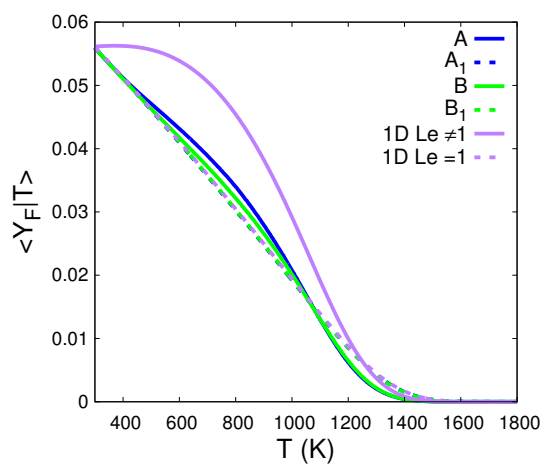

(a) Cases A and B $\left(T_{u}=298 \mathrm{~K}\right)$

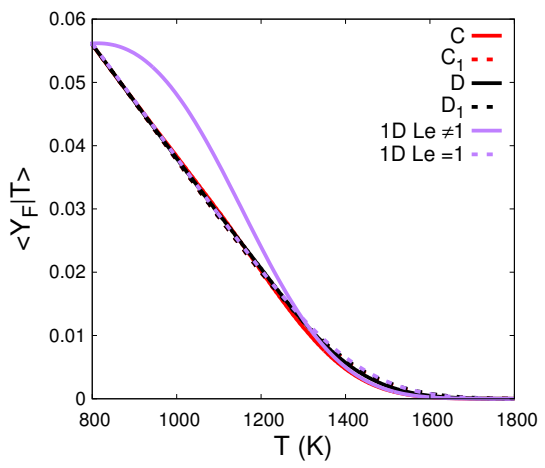

(b) Cases $\mathrm{C}$ and $\mathrm{D}\left(T_{u}=800 \mathrm{~K}\right)$

Fig. 5. Conditional means of fuel mass fractions. 


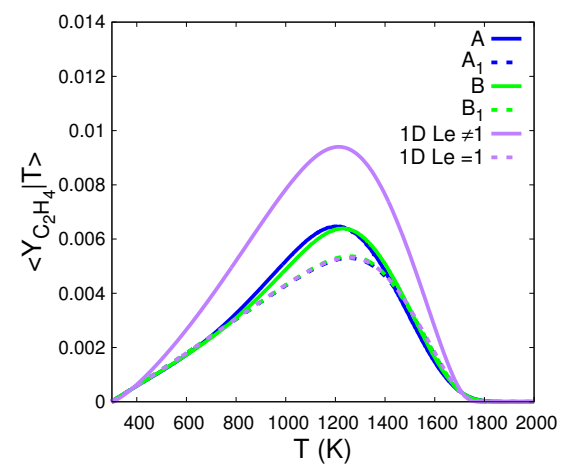

(a) Cases A and B $\left(T_{u}=298 \mathrm{~K}\right)$

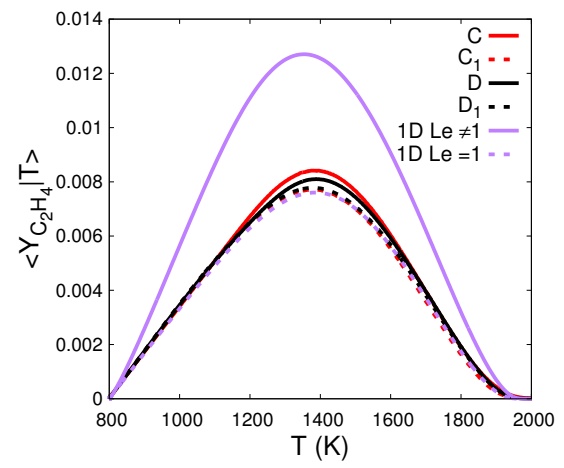

(b) Cases $\mathrm{C}$ and $\mathrm{D}\left(T_{u}=800 \mathrm{~K}\right)$

Fig. 6. Conditional mean of $\mathrm{C}_{2} \mathrm{H}_{4}$ mass fractions. 


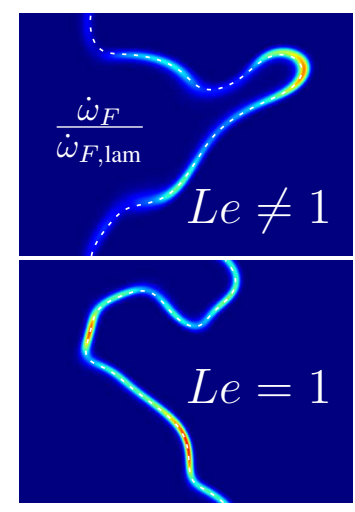

(a) Case A

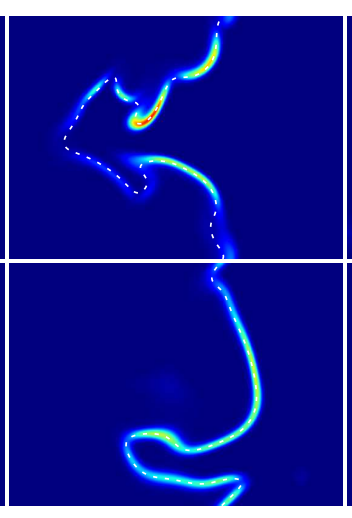

(b) Case B

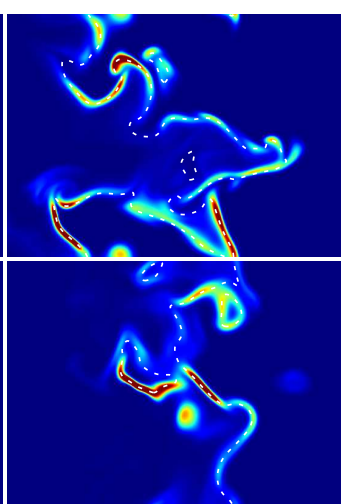

(c) Case C

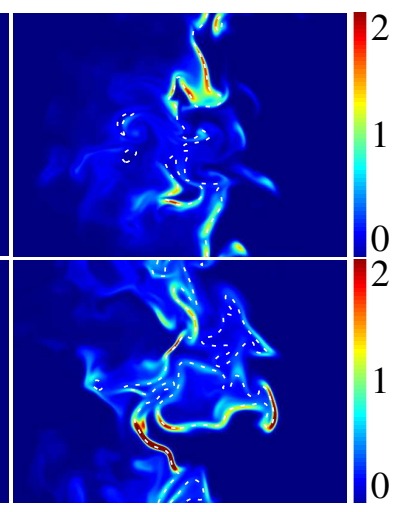

(d) Case D

Fig. 7. Two-dimensional slices of the fuel consumption rate (normalized by the peak value of the corresponding laminar flame). The fuel consumption rate range is satured at $[0,2]$ in each case. The isotherm of peak source term is also shown (white). The top figures correspond to the non-unity Lewis number simulations while the bottom figures correspond to the unity Lewis number cases. 


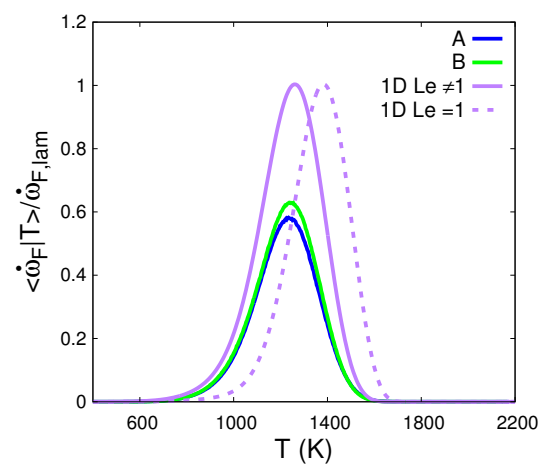

(a) Cases A and B

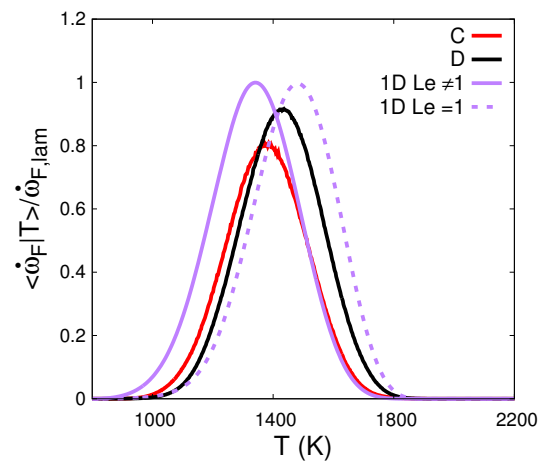

(b) Cases C and D

Fig. 8. Conditional means of normalized fuel consumption rate for non-unity Lewis number simulations. The fuel consumption rates are normalized by the peak value in the corresponding laminar flame. 


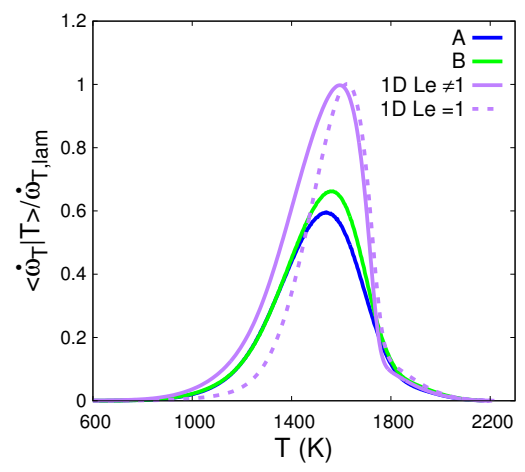

(a) Cases A and B

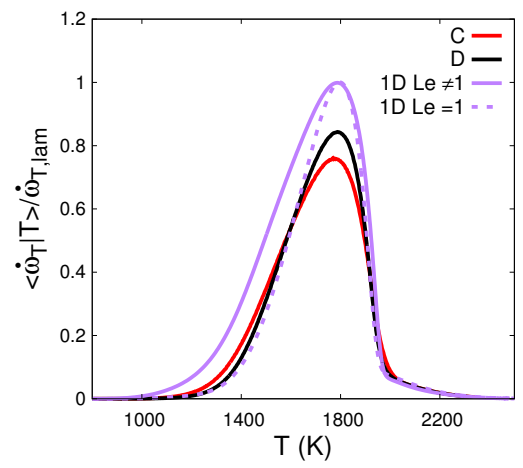

(b) Cases C and D

Fig. 9. Conditional means of normalized heat release rate for non-unity Lewis number simulations. The heat release rates are normalized by the peak value in the corresponding laminar flame. 


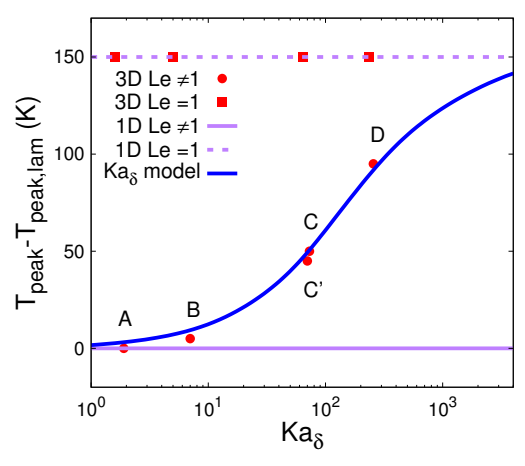

(a) $T_{\text {peak }}$

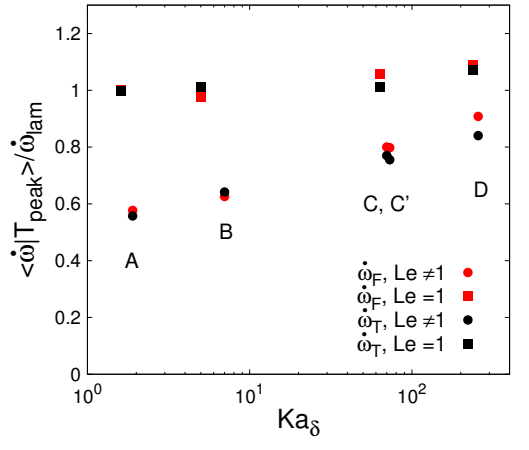

(b) Conditional means

Fig. 10. Temperature at which the peak burning rate is observed for all cases in (a). Conditional means of the normalized fuel consumption and heat release rates at the corresponding temperature of peak source term in (b). Values are normalized by the corresponding laminar flame. 


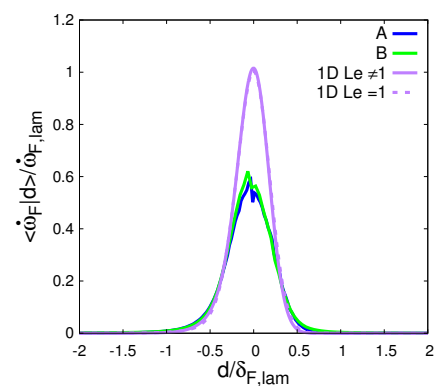

(a) Cases A and B

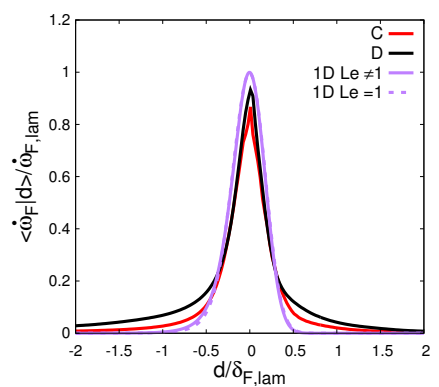

(b) Cases C and D

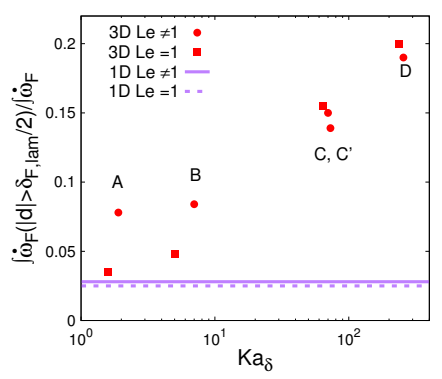

(c) $\% \dot{\omega}_{F}\left(|d|>\delta_{F, \operatorname{lam}} / 2\right)$

Fig. 11. Conditional mean of normalized fuel consumption rate as a function of distance from the peak temperature for the non-unity Lewis number simulations in (a) and (b) and fraction of the total burning rate occuring at $|d|>\delta_{F, \text { lam }} / 2$ for all cases in (c). 


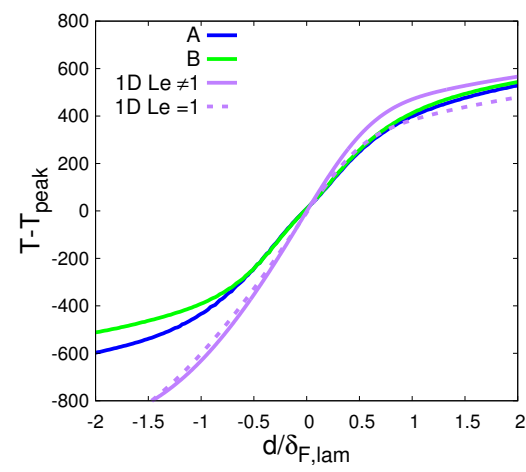

(a) Cases A and B

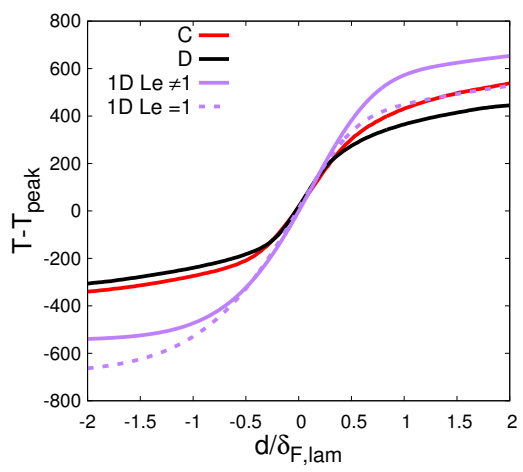

(b) Cases C and D

Fig. 12. Conditional mean of temperature as a function of distance from the peak temperature for the non-unity Lewis number simulations. 


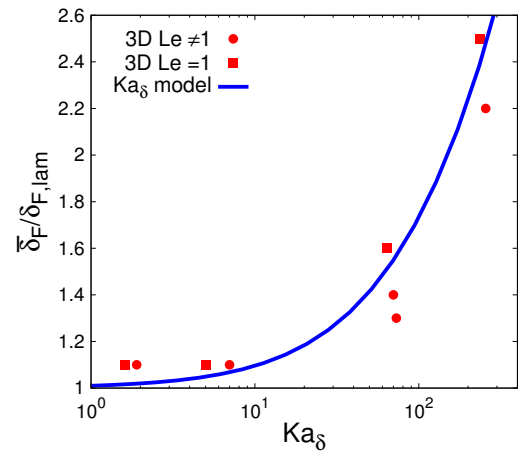

Fig. 13. Normalized fuel reaction zone thickness in physical space. 


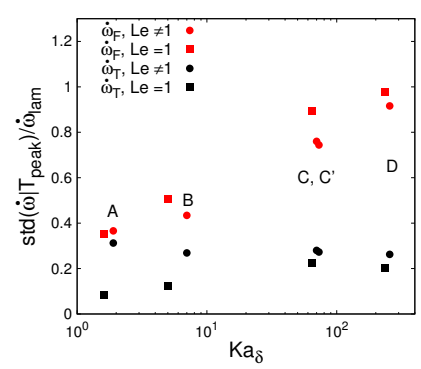

(a) Standard deviations

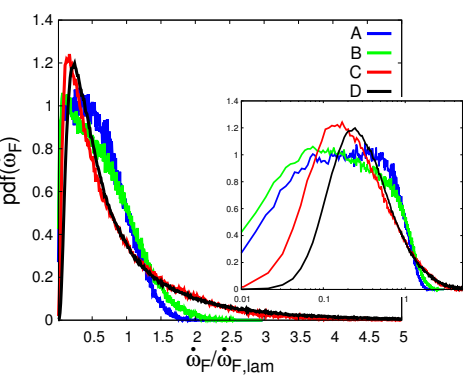

(b) pdf of $\dot{\omega}_{F}$

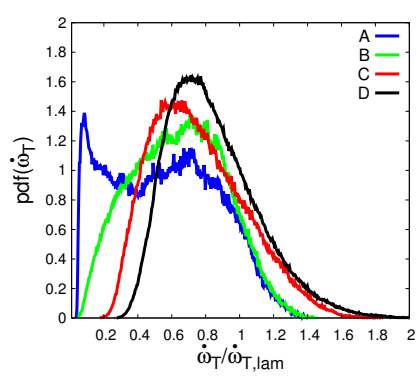

(c) pdf of $\dot{\omega}_{T}$

Fig. 14. Standard deviations of the normalized fuel consumption and heat release rates in (a). Probability density function of the normalized fuel consumption rate in (b). Probability density function of the normalized heat release rate in (c). All taken at the corresponding peak temperature. 


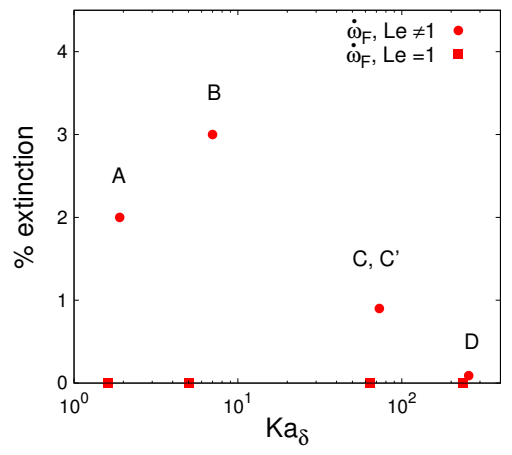

Fig. 15. Probability of local extinction for the different cases. 


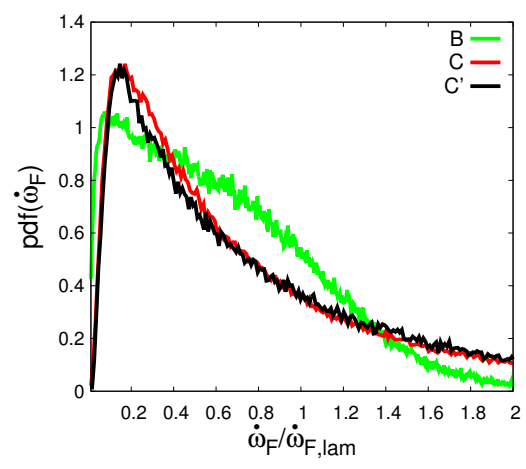

(a) pdf of $\dot{\omega}_{F}$

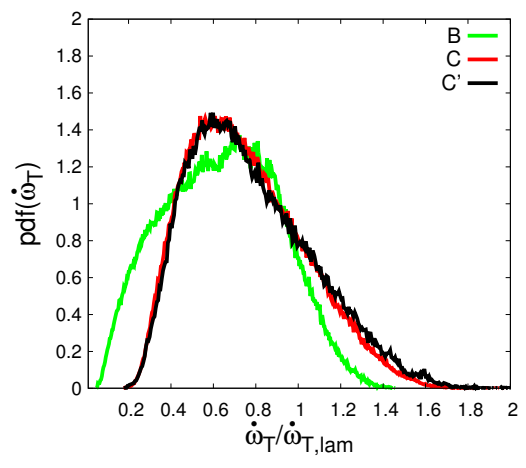

(b) pdf of $\dot{\omega}_{T}$

Fig. 16. Probability density function of the normalized fuel consumption and heat release rates at the corresponding $T_{\text {peak }}$. 


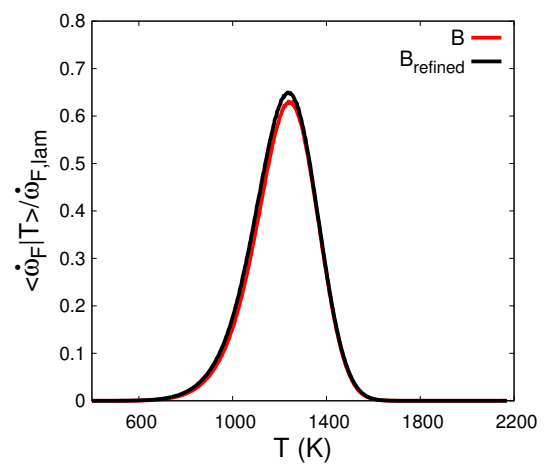

(a) Conditional mean

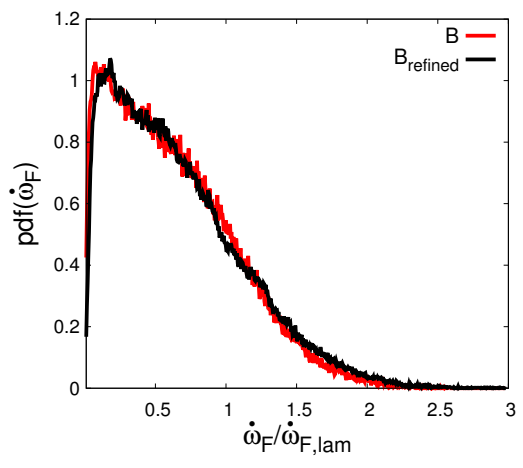

(b) pdf of $\dot{\omega}_{F}$

Fig. 17. Conditional mean and probability density function of the normalized fuel consumption rates. The probability density function was taken at the corresponding temperature of peak fuel consumption. 


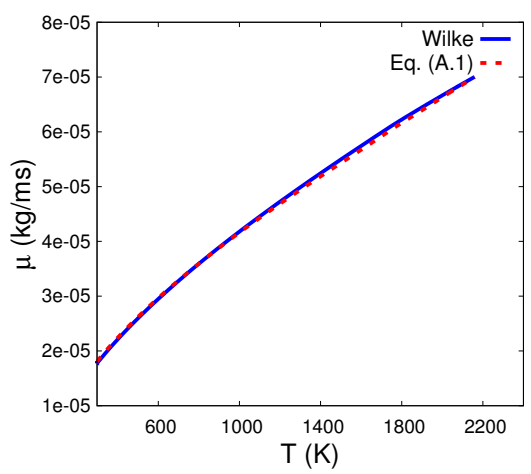

Fig. 18. Mixture-averaged dynamic viscosity of laminar $n$-heptane/air flame computed using Wilke's formula and Eq. (B.1). 


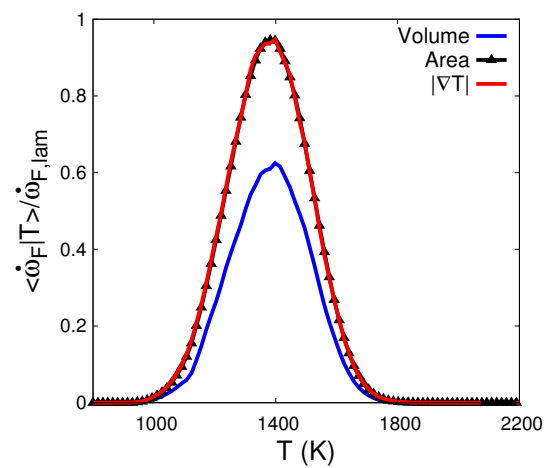

Fig. 19. Conditional means of fuel consumption rate of case $\mathrm{C}$ calculated using volume weighting, surface area weighting, and weighting by the norm of the gradient of temperature. 


\section{List of Tables}

1 Parameters of the simulations. Subscript 1 denotes simulations with unity Lewis numbers. $\phi$ is the equivalence ratio, $S_{L}$ is the laminar flame speed, $l_{F}=\left(T_{b}-T_{u}\right) /|\nabla T|_{\max }$ is the laminar flame thickness, $l=u^{\prime 3} / \epsilon$ is the integral length scale, $\mathrm{Ka}_{u}$ is the Karlovitz number in the unburnt gas, $\mathrm{Ka}_{\delta}$ is the reaction zone Karlovitz number, $\operatorname{Re}_{t}=u^{\prime} l / \nu$ is the turbulent Reynolds number in the unburnt gas, $\eta_{u}$ is the Kolmogorov length scale in the unburnt gas, $\Delta x$ is the grid spacing, $L$ is the domain width and corresponds to $\sim 5 l, T_{\text {peak,lam }}$ is the temperature of peak fuel consumption rate in the corresponding laminar flame, and $T_{\mathrm{ad}}$ is the adiabatic temperature in the corresponding laminar flame.

2 Species Lewis numbers used in the turbulent flame simulations.

3 Time average and standard deviation of the turbulent flame speed as well as fuel effective reaction zone thickness for the different cases.

4 Percentage of contribution of different reactions to the overall fuel consumption rate (first three reactions) and heat release rate (last two reactions) of the corresponding flame.

5 Time average of the normalized turbulent flame speeds, mean fuel consumption rates at $T_{\text {peak }}$, and turbulent flame surface area at $T_{\text {peak }}$ for the different cases.

6 Normalized fuel reaction zone thickness in temperature space and physical space. $\Delta T_{F, \text { lam }}=679 \mathrm{~K}$ for cases $\mathrm{A}$ and $\mathrm{B}$ and $690 \mathrm{~K}$ for cases $\mathrm{C}$ and D. $\delta_{F, \text { lam }}=1.5 \times 10^{-4} \mathrm{~m}$ for cases $\mathrm{A}$ and $\mathrm{B}$ and $1.2 \times 10^{-4} \mathrm{~m}$ for cases $\mathrm{C}$ and $\mathrm{D}$. 


\begin{tabular}{|c|c|c|c|c|c|c|c|c|c|}
\hline Case & A & $\mathrm{A}_{1}$ & $\mathrm{~B}[20]$ & $\mathrm{B}_{1}[20]$ & $\mathrm{C}$ & $\mathrm{C}_{1}$ & $\mathrm{C}^{\prime}$ & $\mathrm{D}$ & $\mathrm{D}_{1}$ \\
\hline$T_{u}(\mathrm{~K})$ & \multicolumn{2}{|c|}{298} & \multicolumn{2}{|c|}{298} & \multicolumn{2}{|c|}{800} & 500 & \multicolumn{2}{|c|}{800} \\
\hline$\phi$ & \multicolumn{2}{|c|}{0.9} & \multicolumn{2}{|c|}{0.9} & \multicolumn{2}{|c|}{0.9} & 0.9 & \multicolumn{2}{|c|}{0.9} \\
\hline$S_{L}(\mathrm{~m} / \mathrm{s})$ & 0.36 & 0.29 & 0.36 & 0.29 & 2.3 & 1.71 & 0.86 & 2.3 & 1.71 \\
\hline$l_{F}(\mathrm{~mm})$ & 0.39 & 0.43 & 0.39 & 0.43 & 0.25 & 0.27 & 0.32 & 0.25 & 0.27 \\
\hline$u^{\prime} / S_{L}$ & 9.0 & 10 & 18 & 21 & 19 & 25 & 38 & 45 & 60 \\
\hline$l / l_{F}$ & 1.1 & 1.0 & 1.1 & 1.0 & 1.2 & 1.1 & 1.0 & 1.2 & 1.1 \\
\hline $\mathrm{Ka}_{u}$ & 78 & 91 & 220 & 280 & 204 & 280 & 648 & 740 & 1050 \\
\hline $\mathrm{Ka}_{\delta}$ & 1.9 & 1.6 & 7.0 & 5.0 & 73 & 64 & 70 & 256 & 237 \\
\hline $\operatorname{Re}_{t}$ & \multicolumn{2}{|c|}{83} & \multicolumn{2}{|c|}{190} & \multicolumn{2}{|c|}{170} & 290 & \multicolumn{2}{|c|}{380} \\
\hline$\eta_{u}(\mathrm{~m})$ & \multicolumn{2}{|c|}{$1.6 \times 10^{-5}$} & \multicolumn{2}{|c|}{$9.0 \times 10^{-6}$} & \multicolumn{2}{|c|}{$7.0 \times 10^{-6}$} & $4.6 \times 10^{-6}$ & \multicolumn{2}{|c|}{$3.5 \times 10^{-6}$} \\
\hline$\Delta x(\mathrm{~m})$ & \multicolumn{2}{|c|}{$1.8 \times 10^{-5}$} & \multicolumn{2}{|c|}{$1.8 \times 10^{-5}$} & \multicolumn{2}{|c|}{$1.2 \times 10^{-5}$} & $1.0 \times 10^{-5}$ & \multicolumn{2}{|c|}{$7.0 \times 10^{-6}$} \\
\hline Domain & \multicolumn{2}{|c|}{$11 L \times L \times L$} & \multicolumn{2}{|c|}{$11 L \times L \times L$} & \multicolumn{2}{|c|}{$11 L \times L \times L$} & $11 L \times L \times L$ & \multicolumn{2}{|c|}{$11 L \times L \times L$} \\
\hline Grid & \multicolumn{2}{|c|}{$11 \times 128^{3}$} & \multicolumn{2}{|c|}{$11 \times 128^{3}$} & \multicolumn{2}{|c|}{$11 \times 128^{3}$} & $11 \times 146^{3}$ & \multicolumn{2}{|c|}{$11 \times 220^{3}$} \\
\hline$T_{\text {peak,lam }}$ & 1240 & 1365 & 1240 & 1365 & 1345 & 1482 & 1290 & 1345 & 1482 \\
\hline$T_{\mathrm{ad}}$ & \multicolumn{2}{|c|}{2188} & \multicolumn{2}{|c|}{2188} & \multicolumn{2}{|c|}{2447} & 2298 & \multicolumn{2}{|c|}{2447} \\
\hline
\end{tabular}

\section{Table 1}

Parameters of the simulations. Subscript 1 denotes simulations with unity Lewis numbers. $\phi$ is the equivalence ratio, $S_{L}$ is the laminar flame speed, $l_{F}=\left(T_{b}-T_{u}\right) /|\nabla T|_{\max }$ is the laminar flame thickness, $l=u^{\prime 3} / \epsilon$ is the integral length scale, $\mathrm{Ka}_{u}$ is the Karlovitz number in the unburnt gas, $\mathrm{Ka}_{\delta}$ is the reaction zone Karlovitz number, $\mathrm{Re}_{t}=u^{\prime} l / \nu$ is the turbulent Reynolds number in the unburnt gas, $\eta_{u}$ is the Kolmogorov length scale in the unburnt gas, $\Delta x$ is the grid spacing, $L$ is the domain width and corresponds to $\sim 5 l, T_{\text {peak,lam }}$ is the temperature of peak fuel consumption rate in the corresponding laminar flame, and $T_{\mathrm{ad}}$ is the adiabatic temperature in the corresponding laminar flame. 


\begin{tabular}{ll|ll|ll}
\hline $\mathrm{N}_{2}$ & 0.99 & $1-\mathrm{CH}_{2}$ & 0.94 & $3-\mathrm{CH}_{2}$ & 0.94 \\
$\mathrm{O}$ & 0.69 & $\mathrm{H}_{2}$ & 0.28 & $\mathrm{H}$ & 0.16 \\
$\mathrm{OH}$ & 0.70 & $\mathrm{H}_{2} \mathrm{O}$ & 0.79 & $\mathrm{O}_{2}$ & 1.06 \\
$\mathrm{HO}_{2}$ & 1.07 & $\mathrm{CH}$ & 0.64 & $\mathrm{CO}$ & 1.07 \\
$\mathrm{HCO}$ & 1.22 & $\mathrm{CH}_{2} \mathrm{O}$ & 1.23 & $\mathrm{CH}_{3}$ & 0.96 \\
$\mathrm{CO}_{2}$ & 1.37 & $\mathrm{CH}_{4}$ & 0.97 & $\mathrm{C}_{2} \mathrm{H}_{3}$ & 1.28 \\
$\mathrm{C}_{2} \mathrm{H}_{4}$ & 1.28 & $\mathrm{C}_{2} \mathrm{H}_{5}$ & 1.39 & $\mathrm{C}_{2} \mathrm{H}$ & 1.25 \\
$\mathrm{HCCO}$ & 0.86 & $\mathrm{C}_{2} \mathrm{H}_{2}$ & 1.27 & $\mathrm{C}_{3} \mathrm{H}_{3}$ & 1.60 \\
$\mathrm{~A}-\mathrm{C}_{3} \mathrm{H}_{5}$ & 1.79 & $n-\mathrm{C}_{3} \mathrm{H}_{7}$ & 1.81 & $\mathrm{C}_{2} \mathrm{H}_{6}$ & 1.40 \\
$\mathrm{P}_{-} \mathrm{C}_{3} \mathrm{H}_{4}$ & 1.68 & $\mathrm{~A}_{-} \mathrm{C}_{3} \mathrm{H}_{4}$ & 1.68 & $\mathrm{C}_{3} \mathrm{H}_{6}$ & 1.80 \\
$1-\mathrm{C}_{4} \mathrm{H}_{8}$ & 1.99 & $1-\mathrm{C}_{5} \mathrm{H}_{10}$ & 2.27 & $1-\mathrm{C}_{5} \mathrm{H}_{11}$ & 2.08 \\
$2-\mathrm{C}_{7} \mathrm{H}_{15}$ & 2.84 & $n-\mathrm{C}_{7} \mathrm{H}_{16}$ & 2.84 & & \\
\hline
\end{tabular}

Table 2

Species Lewis numbers used in the turbulent flame simulations. 
Table 3

\begin{tabular}{c|c|c|c|c|c|c|}
\multirow{2}{*}{ Case } & \multicolumn{2}{|c|}{$\overline{S_{T}} / S_{L}$} & \multicolumn{2}{c|}{$\operatorname{std}\left(S_{T}\right) / S_{L}$} & \multicolumn{2}{c|}{$\overline{\delta_{T}} / \delta_{F, \text { lam }}$} \\
\cline { 2 - 7 } & $\mathrm{Le} \neq 1$ & $\mathrm{Le}=1$ & $\mathrm{Le} \neq 1$ & $\mathrm{Le}=1$ & $\mathrm{Le} \neq 1$ & $\mathrm{Le}=1$ \\
\hline $\mathrm{A}$ & 1.6 & 3.0 & 0.4 & 0.8 & 2.3 & 3.1 \\
$\mathrm{~B}$ & 2.2 & 3.4 & 0.6 & 0.6 & 3.0 & 3.3 \\
$\mathrm{C}$ & 3.3 & 4.6 & 0.7 & 0.9 & 4.4 & 5.5 \\
$\mathrm{C}^{\prime}$ & 3.4 & & 0.6 & & 4.5 & \\
$\mathrm{D}$ & 4.7 & 6.7 & 1.2 & 1.7 & 6.4 & 7.0 \\
\hline
\end{tabular}

Time average and standard deviation of the turbulent flame speed as well as fuel effective reaction zone thickness for the different cases. 


\begin{tabular}{|c|c|c|c|c|c|}
\hline \multicolumn{2}{|c|}{ Reaction/Case } & \multicolumn{2}{c|}{$298 \mathrm{~K}$} & \multicolumn{2}{c|}{$800 \mathrm{~K}$} \\
\cline { 3 - 6 } & & $1 \mathrm{D}$ & Case A & $1 \mathrm{D}$ & Case D \\
\hline \multirow{2}{*}{$n-\mathrm{C}_{7} \mathrm{H}_{16}+\mathrm{H}$} & $\mathrm{Le} \neq 1$ & 59 & 56 & 65 & 68 \\
& $\mathrm{Le}=1$ & 52 & 55 & 55 & 56 \\
\hline \multirow{2}{*}{$n-\mathrm{C}_{7} \mathrm{H}_{16}+\mathrm{OH}$} & $\mathrm{Le} \neq 1$ & 32 & 34 & 26 & 28 \\
& $\mathrm{Le}=1$ & 33 & 31 & 29 & 28 \\
\hline \multirow{2}{*}{$n-\mathrm{C}_{7} \mathrm{H}_{16}+\mathrm{O}$} & $\mathrm{Le} \neq 1$ & 8 & 10 & 8 & 12 \\
& $\mathrm{Le}=1$ & 12 & 13 & 12 & 14 \\
\hline \hline \multirow{2}{*}{$\mathrm{CH}_{3}+\mathrm{O}$} & $\mathrm{Le} \neq 1$ & 12 & 13 & 12 & 13 \\
& $\mathrm{Le}=1$ & 13 & 13 & 13 & 14 \\
\hline \multirow{2}{*}{$\mathrm{HCO}+\mathrm{H}$} & $\mathrm{Le} \neq 1$ & 7 & 6 & 9 & 9 \\
& $\mathrm{Le}=1$ & 9 & 9 & 10 & 10 \\
\hline
\end{tabular}

Table 4

Percentage of contribution of different reactions to the overall fuel consumption rate (first three reactions) and heat release rate (last two reactions) of the corresponding flame. 
Table 5

\begin{tabular}{c|c|c|c|c|c|c|}
\multirow{2}{*}{ Case } & \multicolumn{2}{|c|}{$\overline{S_{T}} / S_{L}$} & \multicolumn{2}{c|}{$<\dot{\omega} \mid T_{\text {peak }}>/ \dot{\omega}_{\text {lam }}$} & \multicolumn{2}{c|}{$\overline{A_{T}} / A$} \\
\cline { 2 - 7 } & Le $\neq 1$ & Le $=1$ & Le $\neq 1$ & Le $=1$ & Le $\neq 1$ & Le $=1$ \\
\hline A & 1.6 & 3.0 & 0.58 & 1.00 & 2.5 & 2.8 \\
B & 2.2 & 3.4 & 0.63 & 0.98 & 3.1 & 3.3 \\
C & 3.3 & 4.6 & 0.80 & 1.06 & 4.3 & 4.2 \\
D & 4.7 & 6.7 & 0.91 & 1.09 & 5.7 & 5.8 \\
\hline
\end{tabular}

Time average of the normalized turbulent flame speeds, mean fuel consumption rates at $T_{\text {peak }}$, and turbulent flame surface area at $T_{\text {peak }}$ for the different cases. 
Table 6

\begin{tabular}{c|c|c|c|c|}
\multirow{2}{*}{ Case } & \multicolumn{2}{|c|}{$\overline{\Delta T_{F}} / \Delta T_{F, \text { lam }}$} & \multicolumn{2}{c|}{$\overline{\delta_{F}} / \delta_{F, \text { lam }}$} \\
\cline { 2 - 5 } & Le $\neq 1$ & Le $=1$ & Le $\neq 1$ & Le $=1$ \\
\hline A & 0.89 & 1.1 & 1.1 & 1.1 \\
B & 0.89 & 1.1 & 1.1 & 1.1 \\
C & 0.90 & 1.0 & 1.3 & 1.6 \\
C $^{\prime}$ & 0.91 & & 1.4 & \\
D & 0.92 & 1.0 & 2.2 & 2.5 \\
\hline
\end{tabular}

Normalized fuel reaction zone thickness in temperature space and physical space. $\Delta T_{F, \text { lam }}=679 \mathrm{~K}$ for cases $\mathrm{A}$ and $\mathrm{B}$ and $690 \mathrm{~K}$ for cases $\mathrm{C}$ and D. $\delta_{F, \mathrm{lam}}=1.5 \times 10^{-4} \mathrm{~m}$ for cases $\mathrm{A}$ and $\mathrm{B}$ and $1.2 \times 10^{-4} \mathrm{~m}$ for cases $\mathrm{C}$ and $\mathrm{D}$. 